\title{
Harmonic Bilocal Fields Generated by Globally Conformal Invariant Scalar Fields
}

\author{
Nikolay M. Nikolov ${ }^{1,2}$, Karl-Henning Rehren ${ }^{3}$, Ivan Todorov ${ }^{1,2}$ \\ 1 Institute for Nuclear Research and Nuclear Energy, Tsarigradsko Chaussee 72, BG-1784 Sofia, \\ Bulgaria. E-mail: mitov@inrne.bas.bg; todorov@inrne.bas.bg \\ 2 Abdus Salam International Centre for Theoretical Physics, Strada Costiera 11, I-34014 Trieste, Italy \\ 3 Institut für Theoretische Physik, Universität Göttingen, Friedrich-Hund-Platz 1, D-37077 Göttingen, \\ Germany. E-mail: rehren@ theorie.physik.uni-goe.de
}

Received: 11 May 2007 / Accepted: 5 June 2007

Published online: 8 February 2008 - (C) The Author(s) 2008

\begin{abstract}
The twist two contribution in the operator product expansion of $\phi_{1}\left(\mathrm{x}_{1}\right) \phi_{2}\left(\mathrm{x}_{2}\right)$ for a pair of globally conformal invariant, scalar fields of equal scaling dimension $d$ in four space-time dimensions is a field $V_{1}\left(\mathrm{x}_{1}, \mathrm{x}_{2}\right)$ which is harmonic in both variables. It is demonstrated that the Huygens bilocality of $V_{1}$ can be equivalently characterized by a "single-pole property" concerning the pole structure of the (rational) correlation functions involving the product $\phi_{1}\left(\mathrm{x}_{1}\right) \phi_{2}\left(\mathrm{x}_{2}\right)$. This property is established for the dimension $d=2$ of $\phi_{1}, \phi_{2}$. As an application we prove that any system of GCI scalar fields of conformal dimension 2 (in four space-time dimensions) can be presented as a (possibly infinite) superposition of products of free massless fields.
\end{abstract}

\section{Introduction}

Global Conformal Invariance (GCI) of Minkowski space Wightman fields yields rationality of correlation functions [14]. This result opens the way for a nonperturbative construction and analysis of GCI models for higher dimensional Quantum Field Theory (QFT), by exploring further implications of the Wightman axioms.

By choosing the axiomatic approach, we avoid any bias about the possible origin of the model, because we aim at a broadest possible perspective. On the other hand, the assumption of GCI limits the analysis to a class of theories that can be parameterized by its (generating) field content and finitely many coefficients for each correlation function (see Sect. 2). As anomalous dimensions under the assumption of GCI are forced to be integral, there is no perturbative approach within this setting, but it is conceivable that a theory with a continuous coupling parameter may exhibit GCI at discrete values (that appear as renormalization group fixed points). An example of this type is provided by the Thirring model: it is locally conformal invariant for any value of the coupling constant $g$ and becomes GCI for positive integer $g^{2}$ [5].

Previous axiomatic treatments of conformal QFT were focussed on the representation theory and harmonic analysis of the conformal group $[6,10]$ as tools for the Operator 
Product Expansion (OPE). The general projective realization of conformal symmetry in QFT was already emphasized in $[16,17]$ and found to constitute a (partial) organization of the OPE. GCI is complementary in that it assumes true representations (trivial covering projection). A necessary condition for this highly symmetric situation is the presence of infinitely many conserved tensor currents (as we shall see in Sect. 3.3).

The first cases studied under the assumption of GCI were theories generated by a scalar field $\phi(\mathrm{x})$ of (low) integral dimension $d>1$. (The case $d=1$ corresponds to a free massless field with a vanishing truncated 4-point function $w_{4}^{\mathrm{tr}}$.) The cases $2 \leqslant d \leqslant 4$, which give rise to non-zero $w_{4}^{\text {tr }}$ were considered in $[12,13,11] .{ }^{1}$

The main purpose in these papers was to study the constraints for the 4-point correlation (= Wightman) functions coming from the Wightman (= Hilbert space) positivity. This was achieved by using the conformal partial wave expansion. An important technical tool in this expansion is the splitting of the OPE into different twist contributions (see (2.10)). Each partial wave gives a nonrational contribution to the complete rational 4point function. It is therefore remarkable that the sum of the leading, twist two, conformal partial waves (corresponding to the contributions of all conserved symmetric traceless tensors in the OPE of basic fields) can be proven in certain cases to be a rational function. This means that the twist two part in the OPE of two fields $\phi$ is convergent in such cases to a bilocal field, $V_{1}\left(\mathrm{x}_{1}, \mathrm{x}_{2}\right)$, which is our first main result in the present paper. Throughout, "bilocal" means Huygens (= space-like and time-like) locality with respect to both arguments. Proving bilocality exploits the bounds on the poles due to Wightman positivity, and the conservation laws for twist two tensors which imply that the bilocal fields are harmonic in both arguments.

Trivial examples of harmonic bilocal fields are given by bilinear free field constructions of the form : $\varphi\left(\mathrm{x}_{1}\right) \varphi\left(\mathrm{x}_{2}\right):,: \bar{\psi}\left(\mathrm{x}_{1}\right) \gamma_{\mu}\left(\mathrm{x}_{1}-\mathrm{x}_{2}\right)^{\mu} \psi\left(\mathrm{x}_{2}\right):$, or $\left(\mathrm{x}_{1}-\mathrm{x}_{2}\right)^{\mu}\left(\mathrm{x}_{1}-\mathrm{x}_{2}\right)^{\nu}$ $: F_{\mu \sigma}\left(\mathrm{x}_{1}\right) F_{\nu}^{\sigma}\left(\mathrm{x}_{2}\right):$. A major purpose of this paper is to explore whether harmonic twist two fields can exist which are not of this form, and whether they can be bilocal. Moreover, we show that the presence of a bilocal field $V_{1}$ completely determines the structure of the theory in the case of a scaling dimension $d=2$. The first step towards the classification of $d=2$ GCI fields was made in [12] where the case of a unique scalar field was considered. Here we extend our study to the most general case of a theory generated by an arbitrary (countable) set of $d=2$ scalar fields. Our second main result states that such fields are always combinations of Wick products of free fields (and generalized free fields).

The paper is organized as follows.

Section 2 contains a review of relevant results concerning the theory of GCI scalar fields.

In Sect. 3 we study conditions for the existence of the harmonic bilocal field $V_{1}\left(\mathrm{x}_{1}, \mathrm{x}_{2}\right)$. We prove that Huygens bilocality of $V_{1}\left(\mathrm{x}_{1}, \mathrm{x}_{2}\right)$ is equivalent to the single pole property (SPP), Definition 3.1, which is a condition on the pole structure of the leading singularities of the truncated correlation functions of $\phi_{1}\left(\mathrm{x}_{1}\right) \phi_{2}\left(\mathrm{x}_{2}\right)$ whose twist expansion starts with $V_{1}\left(\mathrm{x}_{1}, \mathrm{x}_{2}\right)$. This nontrivial condition qualifies a premature announcement in [2] that Huygens bilocality is automatic.

Indeed, the SPP is trivially satisfied for all correlations of free field constructions of harmonic fields with other (products of) free fields, due to the bilinear structure of $V_{1}$. Thus any violation of the SPP is a clear signal for a nontrivial field content of the model.

1 The last two references are chiefly concerned with the case $d=4$ (in $D=4$ space-time dimensions) which appears to be of particular interest as corresponding to a (gauge invariant) Lagrangian density. The intermediate case $d=3$ is briefly surveyed in [19]. 
Moreover, the SPP will be proven from general principles for an arbitrary system of $d=2$ scalar fields (the case studied in [2]). Yet, although the pole structure of $U\left(\mathrm{x}_{1}, \mathrm{x}_{2}\right)$ turns out to be highly constrained in general by the conservation laws of twist two tensor currents, the SPP does not follow for fields of higher dimensions, as illustrated by a counter-example of a 6-point function of $d=4$ scalar fields involving double poles (Sect. 3.5).

The existence of $V_{1}\left(\mathrm{x}_{1}, \mathrm{x}_{2}\right)$ in a theory of dimension $d=2$ fields allows to determine the truncated correlation functions up to a single parameter in each of them. This is exploited in Sect. 4, where an associative algebra structure of the OPE of $d=2$ scalar fields and harmonic bilocal fields is revealed. The free-field representation of these fields is inferred by solving an associated moment problem.

\section{Properties of GCI Scalar Fields}

2.1. Structure of correlation functions and pole bounds. We assume throughout the validity of the Wightman axioms for a QFT on the $D=4$ flat Minkowski space-time $M$ (except for asymptotic completeness) - see [18]. Our results can be, in fact, generalized in a straightforward way to any even space-time dimension $D$. The condition of GCI in the Minkowski space is an additional symmetry condition on the correlation functions of the theory [14]. In the case of a scalar field $\phi(\mathrm{x})$, it asserts that the correlation functions of $\phi(\mathrm{x})$ are invariant under the substitution

$$
\phi(\mathrm{x}) \mapsto \operatorname{det}\left(\frac{\partial g}{\partial \mathrm{x}}\right)^{\frac{d}{4}} \phi(g(\mathrm{x})),
$$

where $\mathrm{x} \mapsto g(\mathrm{x})$ is any conformal transformation of the Minkowski space, $\frac{\partial g}{\partial \mathrm{x}}$ is its Jacobi matrix and $d>0$ is the scaling dimension of $\phi$. An important point is that the invariance of Wightman functions $\left\langle 0\left|\phi\left(\mathrm{x}_{1}\right) \cdots \phi\left(\mathrm{x}_{n}\right)\right| 0\right\rangle$ under the transformation (2.1) should be valid for all $\mathrm{x}_{k} \in M$ in the domain of definition of $g$ (in the sense of distributions). It follows that $d$ must be an integer in order to ensure the singlevaluedness of the prefactor in (2.1). Thus, GCI implies that only integral anomalous dimensions can occur.

The most important consequences of GCI in the case of scalar fields $\phi_{k}(\mathrm{x})$ of dimensions $d_{k}$ are summarized as follows:

(a) Huygens Locality ([14, Theorem 4.1]). Fields commute for non light-like separations. This has an algebraic version:

$$
\left[\left(\mathrm{x}_{1}-\mathrm{x}_{2}\right)^{2}\right]^{N}\left[\phi_{1}\left(\mathrm{x}_{1}\right), \phi_{2}\left(\mathrm{x}_{2}\right)\right]=0
$$

for a sufficiently large integer $N$.

(b) Rationality of Correlation Functions (cf. [14, Theorem 3.1]). The general form of Wightman functions is:

$$
\left\langle 0\left|\phi_{1}\left(\mathrm{x}_{1}\right) \cdots \phi_{n}\left(\mathrm{x}_{n}\right)\right| 0\right\rangle=\sum_{\left\{\mu_{j k}\right\}} C_{\left\{\mu_{j k}\right\}} \prod_{j<k}\left(\rho_{j k}\right)^{\mu_{j k}},
$$

where here and in what follows we set

$$
\rho_{j k}:=\left(\mathrm{x}_{j k}-i 0 \mathrm{e}_{0}\right)^{2}=\left(\mathrm{x}_{j k}\right)^{2}+i 0 \mathrm{x}_{j k}^{0}, \quad \mathrm{x}_{j k}:=\mathrm{x}_{j}-\mathrm{x}_{k} ;
$$


the sum in Eq. (2.3) is over all configurations of integral powers $\left\{\mu_{j k}=\mu_{k j}\right\}$ subject to the following conditions:

$$
\sum_{j(\neq k)} \mu_{j k}=-d_{k}
$$

and pole bounds $\mu_{j k} \geqslant-\llbracket \frac{d_{j}+d_{k}}{2}+\frac{\delta_{d_{j} d_{k}}-1}{2} \rrbracket$. Equation (2.5) follows from the conformal invariance under (2.1); the pole bounds express the absence of non-unitary representations in the OPE of two fields [14, Lemma 4.3]. Under these conditions the sum in (2.3) is always finite and there are a finite number of free parameters for every $n$-point correlation function. We shall refer to the form (2.3) as a Laurent polynomial in the variables $\rho_{j k}{ }^{2}$

(c) The truncated Wightman functions $\left\langle 0\left|\phi_{1}\left(\mathrm{x}_{1}\right) \cdots \phi_{n}\left(\mathrm{x}_{n}\right)\right| 0\right\rangle^{\mathrm{tr}}$ are of the same form like (2.3) but with pole degrees $\mu_{j k}^{\mathrm{tr}}$ bounded by

$$
\mu_{j k}^{\mathrm{tr}}>-\frac{d_{j}+d_{k}}{2}
$$

(cf. [14, Corollary 4.4]).

The cluster condition, expressing the uniqueness of the vacuum, requires that if a non-empty proper subset of points $\mathrm{x}_{k}$ among all $\mathrm{x}_{i}(i=1, \ldots, n)$ is shifted by $t \cdot \mathrm{a}$ $\left(\mathrm{a}^{2} \neq 0\right)$, then the truncated function must vanish in the limit $t \rightarrow \infty$. For the two-point clusters $\left\{\mathrm{x}_{j}, \mathrm{x}_{k}\right\}$, this condition is ensured by (2.6) in combination with (2.5). For higher clusters, it puts further constraints on the admissible linear combinations of terms of the form (2.3). Note however, that because of possible cancellations the individual terms need not vanish in the cluster limit.

The cluster condition will be used in establishing the single pole property for $d=2$.

2.2. Twist expansion of the OPE and bi-harmonicity of twist two contribution. The most powerful tool provided by GCI is the explicit construction of the OPE of local fields in the general (axiomatic) framework.

Let $\phi_{1}(\mathrm{x})$ and $\phi_{2}(\mathrm{x})$ be two GCI scalar fields of the same scaling dimension $d$ and consider the operator distribution

$$
U\left(\mathrm{x}_{1}, \mathrm{x}_{2}\right)=\left(\rho_{12}\right)^{d-1}\left(\phi_{1}\left(\mathrm{x}_{1}\right) \phi_{2}\left(\mathrm{x}_{2}\right)-\left\langle 0\left|\phi_{1}\left(\mathrm{x}_{1}\right) \phi_{2}\left(\mathrm{x}_{2}\right)\right| 0\right\rangle\right) .
$$

As a consequence of the pole bounds (2.6), $U\left(\mathrm{x}_{1}, \mathrm{x}_{2}\right)$ is smooth in the difference $\mathrm{x}_{12}$. This is to be understood in a weak sense for matrix elements of $U$ between bounded energy states. Obviously, $U\left(\mathrm{x}_{1}, \mathrm{x}_{2}\right)$ is a Huygens bilocal field in the sense that

$$
\left[\left(\mathrm{x}_{1}-\mathrm{x}\right)^{2}\left(\mathrm{x}_{2}-\mathrm{x}\right)^{2}\right]^{N}\left[U\left(\mathrm{x}_{1}, \mathrm{x}_{2}\right), \psi(\mathrm{x})\right]=0
$$

for every field $\psi(\mathrm{x})$ that is Huygens local with respect to $\phi_{k}(\mathrm{x})$. Then, one introduces the OPE of $\phi_{1}\left(\mathrm{x}_{1}\right) \phi_{2}\left(\mathrm{x}_{2}\right)$ by the Taylor expansion of $U$ in $\mathrm{x}_{12}$,

$$
U\left(\mathrm{x}_{1}, \mathrm{x}_{2}\right)=\sum_{n=0}^{\infty} \sum_{\mu_{1}, \ldots, \mu_{n}=0}^{3} \mathrm{x}_{12}^{\mu_{1}} \cdots \mathrm{x}_{12}^{\mu_{n}} X_{\mu_{1} \ldots \mu_{n}}^{n}\left(\mathrm{x}_{2}\right),
$$

\footnotetext{
2 Writing correlation functions in terms of the conformally invariant cross ratios is particularly useful to parameterize 4-point functions. A basis of cross ratios for an $n$-point function is used in the proof of Lemma 3.6. The general systematics of the pole structure, however, is more transparent in terms of the present variables.
} 
where $X_{\mu_{1} \ldots \mu_{n}}^{n}\left(\mathrm{x}_{2}\right)$ are Huygens local fields. We can consider the series (2.9) as a formal power series, or as a convergent series in terms of the analytically continued correlation functions of $U\left(\mathrm{x}_{1}, \mathrm{x}_{2}\right)$. We will consider at this point the series (2.9) just as a formal series. (See also [1] for the general case of constructing OPE via multilocal fields in the context of vertex algebras in higher dimensions.)

Since the prefactor in (2.7) transforms as a scalar density of conformal weight (1 $d, 1-d)$ then $U\left(\mathrm{x}_{1}, \mathrm{x}_{2}\right)$ transforms as a conformal bilocal field of weight $(1,1)$. Hence, the local fields $X_{\mu_{1} \ldots \mu_{n}}^{n}$ in (2.9) have scaling dimensions $n+2$ but are not, in general, quasiprimary. ${ }^{3}$ One can pass to an expansion in quasiprimary fields by subtracting from $X_{\mu_{1} \ldots \mu_{n}}^{n}$ derivatives of lower dimensional fields $X_{\mu_{1} \ldots \mu_{n^{\prime}}}^{n^{\prime}}$. The resulting quasiprimary fields $O_{\mu_{1} \ldots \mu_{\ell}}^{k}$ are traceless tensor fields of rank $\ell$ and dimension $k$. The difference

$$
k-\ell \text { ("dimension - rank") }
$$

is called twist of the tensor field $O_{\mu_{1} \ldots \mu_{\ell}}^{k}$. Unitarity implies that the twist is non-negative [10], and by GCI, it should be an even integer. In this way one can reorganize the OPE (2.9) as follows:

$$
U\left(\mathrm{x}_{1}, \mathrm{x}_{2}\right)=V_{1}\left(\mathrm{x}_{1}, \mathrm{x}_{2}\right)+\rho_{12} V_{2}\left(\mathrm{x}_{1}, \mathrm{x}_{2}\right)+\left(\rho_{12}\right)^{2} V_{3}\left(\mathrm{x}_{1}, \mathrm{x}_{2}\right)+\cdots,
$$

where $V_{\kappa}\left(\mathrm{x}_{1}, \mathrm{x}_{2}\right)$ is the part of the OPE (2.9) containing only twist $2 \kappa$ contributions. Note that Eq. (2.11) contains also the information that the twist $2 \kappa$ contributions contain a factor $\left(\rho_{12}\right)^{\kappa-1}$ (i.e. $V_{\kappa}$ are "regular" at $\mathrm{x}_{1}=\mathrm{x}_{2}$ ), which is a nontrivial feature of this OPE (obtained by considering 3-point functions). Thus, the expansion in twists can be viewed as a light-cone expansion of the OPE.

Since the twist decomposition of the fields is conformally invariant then each $V_{\kappa}$ will behave, at least infinitesimally, as a scalar $(\kappa, \kappa)$ density under conformal transformations.

Every $V_{\kappa}$ is a complicated (formal) series in twist $2 \kappa$ fields and their derivatives:

$$
V_{\kappa}\left(\mathrm{x}_{1}, \mathrm{x}_{2}\right)=\sum_{\ell=0}^{\infty} K_{\kappa}^{\mu_{1} \ldots \mu_{\ell}}\left(\mathrm{x}_{12}, \partial_{\mathrm{x}_{2}}\right) O_{\mu_{1} \ldots \mu_{\ell}}^{\ell+2 \kappa}\left(\mathrm{x}_{2}\right),
$$

where $K_{\kappa}^{\mu_{1} \ldots \mu_{\ell}}\left(\mathrm{x}_{12}, \partial_{\mathrm{x}_{2}}\right)$ are infinite formal power series in $\mathrm{x}_{12}$ with coefficients that are differential operators in $\mathrm{x}_{2}$ acting on the quasiprimary fields $O$. The important point here is that the series $K_{\kappa}^{\mu_{1} \ldots \mu_{\ell}}\left(\mathrm{x}_{12}, \partial_{\mathrm{x}_{2}}\right)$ can be fixed universally for any (even generally) conformal QFT. This is due to the universality of conformal 3-point functions. The explicit form of $K_{\kappa}^{\mu_{1} \ldots \mu_{\ell}}\left(\mathrm{x}_{12}, \partial_{\mathrm{x}_{2}}\right)$ can be found in [6,7] (see also [13]).

Thus, we can at this point consider $V_{\kappa}\left(\mathrm{x}_{1}, \mathrm{x}_{2}\right)$ only as generating series for the twist $2 \kappa$ contributions to the OPE of $\phi\left(\mathrm{x}_{1}\right) \phi\left(\mathrm{x}_{2}\right)$ but we still do not know whether these series would be convergent and even if they were, it would not be evident whether they would give bilocal fields. In the next section we will see that this is true for the leading, twist two part under certain conditions, which are automatically fulfilled for $d=2$.

The higher twist parts $V_{\kappa}(\kappa>1)$ are certainly not convergent to Huygens bilocal fields, since their 4-point functions, computed in [13], are not rational.

The major difference between the twist two tensor fields and the higher twist fields is that the former satisfy conservation laws:

$$
\partial_{x_{\mu_{1}}} O_{\mu_{1} \ldots \mu_{\ell}}^{\ell+2}(\mathrm{x})=0 \quad(\ell \geqslant 1) .
$$

\footnotetext{
3 Quasiprimary fields transform irreducibly under conformal transformations.
} 
This is a well known consequence of the conformal invariance of the 2-point function and the Reeh-Schlieder theorem. It includes, in particular, the conservation laws of the currents and the stress-energy tensor. It turns out that $V_{1}\left(\mathrm{x}_{1}, \mathrm{x}_{2}\right)$ encodes in a simple way this infinite system of equations.

Theorem 2.1. ([13]). The system of differential equations (2.13) is equivalent to the harmonicity of $V_{1}\left(\mathrm{x}_{1}, \mathrm{x}_{2}\right)$ in both arguments (bi-harmonicity) as a formal series, i.e.,

$$
\square_{\mathrm{x}_{1}} V_{1}\left(\mathrm{x}_{1}, \mathrm{x}_{2}\right)=0=\square_{\mathrm{x}_{2}} V_{1}\left(\mathrm{x}_{1}, \mathrm{x}_{2}\right)
$$

The proof is based on the explicit knowledge of the $K$ series in (2.12) and it is valid even if the theory is invariant under infinitesimal conformal transformations only.

The separation of the twist two part in (2.11) amounts to a splitting of $U$ of the form

$$
U\left(\mathrm{x}_{1}, \mathrm{x}_{2}\right)=V_{1}\left(\mathrm{x}_{1}, \mathrm{x}_{2}\right)+\rho_{12} \widetilde{U}\left(\mathrm{x}_{1}, \mathrm{x}_{2}\right) .
$$

This splitting can be thought of in terms of matrix elements of $U\left(\mathrm{x}_{1}, \mathrm{x}_{2}\right)$ expanded as a formal power series according to (2.9). It is unique by virtue of Theorem 2.1, due to the following classical lemma:

Lemma 2.2. ([3,1]). Let $u(\mathrm{x})$ be a formal power series in $\mathrm{x} \in \mathbb{C}^{4}\left(\right.$ or, $\left.\mathbb{C}^{D}\right)$ with coefficients in a vector space $V$. Then there exist unique formal power series $v(\mathrm{x})$ and $\widetilde{u}(\mathrm{x})$ with coefficients in $V$ such that

$$
u(\mathrm{x})=v(\mathrm{x})+\mathrm{x}^{2} \widetilde{u}(\mathrm{x})
$$

and $v(\mathrm{x})$ is harmonic in $\mathrm{x}$ (i.e., $\square_{\mathrm{x}} v(\mathrm{x})=0$ ). Equation (2.15) is called the harmonic decomposition of $u(\mathrm{x})$ (in the variable $\mathrm{x}$ around $\mathrm{x}=0$ ), and the formal power series $v(\mathrm{x})$ is said to be the harmonic part of $u(\mathrm{x})$.

\section{Bilocality of Twist Two Contribution to the OPE}

Let us sketch our strategy for studying bilocality of $V_{1}\left(\mathrm{x}_{1}, \mathrm{x}_{2}\right)$.

The existence of the field $V_{1}\left(\mathrm{x}_{1}, \mathrm{x}_{2}\right)$ can be established by constructing its correlation functions. On the other hand, every correlation function $\left.4 \cdot V_{1}\left(\mathrm{x}_{1}, \mathrm{x}_{2}\right) \cdot\right\rangle$ of $V_{1}$ is obtained (originally, as a formal power series in $\mathrm{x}_{12}$ ) under the splitting (2.14). It thus appears as a harmonic decomposition of the corresponding correlation function $\left\langle\cdot U\left(\mathrm{x}_{1}, \mathrm{x}_{2}\right) \cdot\right\rangle$ of $U$ :

$$
\left\langle\cdot U\left(\mathrm{x}_{1}, \mathrm{x}_{2}\right) \cdot\right\rangle=\left\langle\cdot V_{1}\left(\mathrm{x}_{1}, \mathrm{x}_{2}\right) \cdot\right\rangle+\rho_{12}\left\langle\cdot \widetilde{U}\left(\mathrm{x}_{1}, \mathrm{x}_{2}\right) \cdot\right\rangle \cdot
$$

Note that we should initially treat the left-hand side of (3.1) also as a formal power series in $\mathrm{x}_{12}$ in order to make the equality meaningful. It is important that this series is always convergent as a Taylor expansion of a rational function in a certain domain around $\mathrm{x}_{1}=\mathrm{x}_{2}$ in $M_{\mathbb{C}}^{\times 2}$, for the complexified Minkowski space $M_{\mathbb{C}}=M+i M$, according to the standard analytic properties of Wightman functions. We shall show in Sect. 3.1

\footnotetext{
4 This short-hand notation stands for $\left\langle 0\left|\phi_{3}\left(\mathrm{x}_{3}\right) \cdots \phi_{k}\left(\mathrm{x}_{k}\right) V_{1}\left(\mathrm{x}_{1}, \mathrm{x}_{2}\right) \phi_{k+1}\left(\mathrm{x}_{k+1}\right) \cdots \phi_{n}\left(\mathrm{x}_{n}\right)\right| 0\right\rangle$, here and in the sequel.
} 
that this implies the separate convergence of both terms in the right-hand side of (3.1). Hence, the key tool in constructing $V_{1}$ are the harmonic decompositions

$$
F\left(\mathrm{x}_{1}, \mathrm{x}_{2}\right)=H\left(\mathrm{x}_{1}, \mathrm{x}_{2}\right)+\rho_{12} \widetilde{F}\left(\mathrm{x}_{1}, \mathrm{x}_{2}\right)
$$

of functions $F\left(\mathrm{x}_{1}, \mathrm{x}_{2}\right)$ that are analytic in certain neighbourhoods of the diagonal $\left\{\mathrm{x}_{1}=\mathrm{x}_{2}\right\}$.

Recall that $H$ in (3.2) is uniquely fixed as the harmonic part of $F$ in $\mathrm{x}_{1}$ around $\mathrm{x}_{2}$, due to Lemma 2.2. This is equivalent to the harmonicity $\square_{\mathrm{x}_{1}} H\left(\mathrm{x}_{1}, \mathrm{x}_{2}\right)=0$. On the other hand, according to Theorem 2.1 we have to consider also the second harmonicity condition on $H, \square_{\mathrm{x}_{2}} H\left(\mathrm{x}_{1}, \mathrm{x}_{2}\right)=0$, i.e., $H$ is the harmonic part in $\mathrm{x}_{2}$ around $\mathrm{x}_{1}$. This leads to some "integrability" conditions for the initial function $F\left(\mathrm{x}_{1}, \mathrm{x}_{2}\right)$, which we study in Sect. 3.2.

Next, to characterize the Huygens bilocality of $V_{1}$, we should have rationality of its correlation functions $\left\langle\cdot V_{1}\left(\mathrm{x}_{1}, \mathrm{x}_{2}\right) \cdot\right\rangle$, which is due to a straightforward extension of the arguments of [14, Theorem 3.1]. But we have started with the correlation functions of $U$, which are certainly rational. Hence, we should study another condition on $U$, namely that its correlation functions have a rational harmonic decomposition. We show in Sect. 3.3 that this is equivalent to a simple condition on the correlation functions of $U$, which we call "Single Pole Property" (SPP).

In this way we establish in Sect. 3.4 that $V_{1}$ always exists as a Huygens bilocal field in the case of scalar fields of dimension $d=2$. However, for higher scaling dimensions one cannot anymore expect that $V_{1}$ is Huygens bilocal in general. This is illustrated by a counter-example, involving the 6-point function of a system of $d=4$ fields, given at the end of Sect. 3.5.

3.1. Convergence of harmonic decompositions. To analyze the existence of the harmonic decomposition of a convergent Taylor series we use the complex integration techniques introduced in [1].

Let $M_{\mathbb{C}}=M+i M$ be the complexification of Minkowski space, which in this subsection is assumed to be $D$-dimensional, and $E=\left\{\mathrm{x}:\left(i x^{0}, x^{1}, \ldots, x^{D-1}\right) \in \mathbb{R}^{D}\right\}$ its Euclidean real submanifold, and $\mathbb{S}^{D-1} \subset E$ the unit sphere in $E$. We denote by $\|\cdot\|$ the Hilbert norm related to the fixed coordinates in $M_{\mathbb{C}}:\|\mathrm{x}\|^{2}:=\left|\mathrm{x}^{0}\right|^{2}+\cdots+\left|\mathrm{x}^{D-1}\right|^{2}$.

Let us also introduce for any $r>0$ a real compact submanifold $M_{r}$ of $M_{\mathbb{C}}$ :

$$
M_{r}=\left\{\zeta \in M_{\mathbb{C}}: \zeta=r e^{i \theta} \mathrm{w}, \vartheta \in[0, \pi], \mathrm{w} \in \mathbb{S}^{D-1}\right\}
$$

(note that $\vartheta \in[\pi, 2 \pi]$ gives another parameterization of $M_{r}$ ). Then there is an integral representation for the harmonic part of a convergent Taylor series.

Lemma 3.1 (cf. [1, Sect. 3.3 and Appendix A]). Let $u(\mathrm{x})$ be a complex formal power series that is absolutely convergent in the ball $\|\mathrm{x}\|<r$, for some $r>0$, to an analytic function $U(\mathrm{x})$. Then the harmonic part $v(\mathrm{x})$ of $u(\mathrm{x})$ (around $\mathrm{x}=0$ ), which is provided by Lemma 2.2, is absolutely convergent for

$$
\left|\mathrm{x}^{2}\right|+2 r\|\mathrm{x}\|<r^{2}
$$


The analytic function $V(\mathrm{x})$ that is the sum of the formal power series $v(\mathrm{x})$ has the following integral representation:

$$
V(\mathrm{x})=\int_{M_{r^{\prime}}} \frac{\left.d^{D} \mathrm{z}\right|_{M_{r^{\prime}}}}{\mathfrak{V}_{1}} \frac{1-\frac{\mathrm{x}^{2}}{\mathrm{z}^{2}}}{\left[(\mathrm{z}-\mathrm{x})^{2}\right]^{\frac{D}{2}}} U(\mathrm{z}), \quad \mathfrak{V}_{1}=\left.\int_{M_{1}} d^{D} \mathrm{z}\right|_{M_{1}}=i \pi\left|\mathbb{S}^{D-1}\right|,
$$

where $r^{\prime}<r,\left|\mathrm{x}^{2}\right|+2 r^{\prime}\|\mathrm{x}\|<r^{\prime 2}$, and the (complex) integration measure $\left.d^{D} \mathrm{z}\right|_{M_{r^{\prime}}}$ is obtained by the restriction of the complex volume form $d^{D} \mathrm{Z}\left(=d z^{0} \wedge \cdots \wedge d z^{D-1}\right)$ on $M_{\mathbb{C}}\left(\cong \mathbb{C}^{D}\right)$ to the real $D$-dimensional submanifold $M_{r^{\prime}}(3.3), r^{\prime}>0$.

Proof. Consider the Taylor expansion in $\mathrm{x}$ of the function $\left(1-\frac{\mathrm{x}^{2}}{\mathrm{z}^{2}}\right) /\left[(\mathrm{z}-\mathrm{x})^{2}\right]^{\frac{D}{2}}$ and write it in the form (cf. [1, Sect. 3.3])

$$
\frac{1-\frac{\mathrm{x}^{2}}{\mathrm{z}^{2}}}{\left[(\mathrm{z}-\mathrm{x})^{2}\right]^{\frac{D}{2}}}=\sum_{\ell=0}^{\infty}\left(\mathrm{z}^{2}\right)^{-\frac{D}{2}-\ell} H_{\ell}(\mathrm{z}, \mathrm{x}), \quad H_{\ell}(\mathrm{z}, \mathrm{x})=\sum_{\mu} h_{\ell \mu}(\mathrm{z}) h_{\ell \mu}(\mathrm{x}),
$$

where $\left\{h_{\ell \mu}(\mathrm{u})\right\}$ is an orthonormal basis of harmonic homogeneous polynomials of degree $\ell$ on the sphere $\mathbb{S}^{D-1}$. This expansion is convergent for

$$
\left|x^{2}\right|+2|z \cdot x|<\left|z^{2}\right|
$$

since its left-hand side is related to the generating function for $H_{\ell}$ :

$$
\frac{1-\lambda^{2} x^{2} y^{2}}{\left(1-2 \lambda x \cdot y+\lambda^{2} x^{2} y^{2}\right)^{\frac{D}{2}}}=\sum_{\ell=0}^{\infty} \lambda^{\ell} H_{\ell}(x, y)
$$

the expansion (3.8) being convergent for $\lambda \leqslant 1$ if $\left|\mathrm{x}^{2} \mathrm{y}^{2}\right|+2|\mathrm{x} \cdot \mathrm{y}|<1$. Then if we fix $r^{\prime}<r$ and $\mathrm{z}$ varies on $M_{r^{\prime}}$, a sufficient condition for (3.7) is $\left|\mathrm{x}^{2}\right|+2 r^{\prime}\|\mathrm{x}\|<r^{\prime 2}$ (since

$\sup |\mathrm{w} \cdot \mathrm{x}|=\|\mathrm{x}\|)$. $\mathrm{w} \in \mathbb{S}^{D-1}$

On the other hand, writing $u(\mathrm{z})=\sum_{k=0}^{\infty} u_{k}(\mathrm{z})$, where $u_{k}$ are homogeneous polynomials of degree $k$, we get by the absolute convergence of $u(\mathrm{z})$ the relation (valid for $\left|\mathrm{x}^{2}\right|$ $\left.+2 r^{\prime}\|\mathrm{x}\|<r^{\prime 2}\right)$

$$
\int_{M_{r^{\prime}}} \frac{\left.d^{D} \mathrm{z}\right|_{M_{r^{\prime}}}}{\mathfrak{V}_{1}} \frac{1-\frac{\mathrm{x}^{2}}{\mathrm{z}^{2}}}{\left[(\mathrm{z}-\mathrm{x})^{2}\right]^{\frac{D}{2}}} U(\mathrm{z})=\sum_{k, \ell=0}^{\infty} \int_{M_{r^{\prime}}} \frac{\left.d^{D} \mathrm{z}\right|_{M_{r^{\prime}}}}{\mathfrak{V}_{1}}\left(\mathrm{z}^{2}\right)^{-\frac{D}{2}-\ell} H_{\ell}(\mathrm{x}, \mathrm{z}) u_{k}(\mathrm{z}) .
$$

Noting next that in the parameterization (3.3) of $M_{r^{\prime}}$ we have $\left.d^{D} \mathrm{z}\right|_{M_{r^{\prime}}}=i r^{\prime D} e^{i D \vartheta}$ $d \vartheta \wedge d \sigma(\mathrm{w})$, where $d \sigma(\mathrm{w})$ is the volume form on the unit sphere, we obtain for the right-hand side of (3.9):

$$
\sum_{k, \ell=0}^{\infty} \int_{0}^{\pi} \frac{d \vartheta}{i \pi} e^{i \vartheta(k-\ell)} \int_{\mathbb{S}^{D-1}} \frac{d \sigma(\mathrm{w})}{\left|\mathbb{S}^{D-1}\right|} H_{\ell}(\mathrm{x}, \mathrm{w}) u_{k}(\mathrm{w}) .
$$


Now if we write, according to Lemma 2.2, $u_{k}(\mathrm{z})=\sum_{2 j \leqslant k} \sum_{\mu^{\prime}} c_{k, j, \mu^{\prime}}\left(\mathrm{z}^{2}\right)^{j} h_{k-2 j, \mu^{\prime}}(\mathrm{z})$, then we get by the orthonormality of $h_{\ell, \mu}(\mathrm{w})$,

$$
\begin{array}{r}
\sum_{k, \ell=0}^{\infty} \sum_{2 j \leqslant k} \sum_{\mu} \delta_{\ell, k-2 j} \int_{0}^{\pi} \frac{d \vartheta}{i \pi} e^{i \vartheta(k-\ell)} c_{k, j, \mu} h_{k-2 j, \mu}(\mathrm{x}) \\
=\sum_{k=0}^{\infty} \sum_{\mu} c_{k, 0, \mu} h_{k, \mu}(\mathrm{x})=v(\mathrm{x}) .
\end{array}
$$

The latter proves both the convergence of $v(\mathrm{x})$ in the domain (3.4) (since $r^{\prime}<r$ was arbitrary) and the integral representation (3.5).

As an application of this result we will prove now

Proposition 3.2. For all $n$ and $k$, and for all local fields $\phi_{j}(j=3, \ldots, n)$ the Taylor series

$$
\left\langle 0\left|\phi_{3}\left(\mathrm{x}_{3}\right) \cdots \phi_{k}\left(\mathrm{x}_{k}\right) V_{1}\left(\mathrm{x}_{1}, \mathrm{x}_{2}\right) \phi_{k+1}\left(\mathrm{x}_{k+1}\right) \cdots \phi_{n}\left(\mathrm{x}_{n}\right)\right| 0\right\rangle
$$

in $\mathrm{x}_{12}$ converge absolutely in the domain

$$
\left(\left\|\mathrm{x}_{12}\right\|+\sqrt{\left\|\mathrm{x}_{12}\right\|^{2}+\left|\mathrm{x}_{12}^{2}\right|}\right)\left(\left\|\mathrm{x}_{2 j}\right\|+\sqrt{\left\|\mathrm{x}_{2 j}\right\|^{2}+\left|\mathrm{x}_{2 j}^{2}\right|}\right)<\left|\mathrm{x}_{2 j}^{2}\right| \quad \forall j
$$

$(j=3, \ldots, n)$. They all are real analytic and independent of $k$ for mutually nonisotropic points.

Proof. Let

$$
\begin{aligned}
& F_{k}\left(\mathrm{x}_{12}, \mathrm{x}_{23}, \ldots, \mathrm{x}_{2 n}\right) \\
& \quad=\left\langle 0\left|\phi_{3}\left(\mathrm{x}_{3}\right) \cdots \phi_{k}\left(\mathrm{x}_{k}\right) U\left(\mathrm{x}_{1}, \mathrm{x}_{2}\right) \phi_{k+1}\left(\mathrm{x}_{k+1}\right) \cdots \phi_{n}\left(\mathrm{x}_{n}\right)\right| 0\right\rangle
\end{aligned}
$$

be the correlation functions, analytically continued in $\mathrm{x}_{12}$.

As $F_{k}$, which is a rational function, depends on $\mathrm{x}:=\mathrm{x}_{12}$ via a sum of products of powers $\left[\left(\mathrm{x}-\mathrm{x}_{2 j}\right)^{2}\right]^{-\mu_{j}}$ it has a convergent expansion in $\mathrm{x}$ for

$$
\left|\mathrm{x}^{2}\right|+2\left|\mathrm{x} \cdot \mathrm{x}_{2 j}\right|<\left|\mathrm{x}_{2 j}^{2}\right| \text {. }
$$

If we want $F_{k}$ to have a convergent Taylor expansion for $\|\mathrm{x}\|<r$ we get the following sufficient condition:

$$
r^{2}<\left|\mathrm{x}_{2 j}^{2}\right|-2 r\left\|\mathrm{x}_{2 j}\right\|
$$

By Lemma 3.1 we conclude that the series (3.10) is convergent for

$$
\left|\mathrm{x}_{12}^{2}\right|+2 r \| \mathrm{x}_{12}||<r^{2} .
$$

Combining both (sufficient) conditions (3.14) and (3.15) for $r$ we find that they are compatible if $\left\|\mathrm{x}_{12}\right\|+\sqrt{\left\|\mathrm{x}_{12}\right\|^{2}+\left|\mathrm{x}_{12}^{2}\right|}<\sqrt{\left\|\mathrm{x}_{2 j}\right\|^{2}+\left|\mathrm{x}_{2 j}^{2}\right|}-\left\|\mathrm{x}_{2 j}\right\|$, which is equivalent to $(3.11)$. 
Note that one can also prove a similar convergence property for the correlation functions of several $V_{1}$.

Remark 3.1. The domain of convergence of (3.10) should be Lorentz invariant. Hence, (3.10) are convergent in the smallest Lorentz invariant set containing the domain (3.11). Such a set is determined by the values of the invariants $\mathrm{x}_{12}^{2}, \mathrm{x}_{2 j}^{2}$ and $\mathrm{x}_{12} \cdot \mathrm{x}_{2 j}$ and it turns out to be the set

$$
\begin{aligned}
& \qquad\left|\mathrm{x}_{12}^{2}\right|^{\frac{1}{2}}\left|\mathrm{x}_{2 j}^{2}\right|^{\frac{1}{2}} \leqslant\left|\mathrm{x}_{12} \cdot \mathrm{x}_{2 j}\right|<\frac{\left(\left|\mathrm{x}_{2 j}^{2}\right|^{\frac{1}{2}}-\left|\mathrm{x}_{12}^{2}\right|^{\frac{1}{2}}\right)^{2}}{4} \\
& \text { or equivalently } \sqrt{\left|\mathrm{x}_{12}^{2}\right|\left|\mathrm{x}_{2 j}^{2}\right|+\left|\mathrm{x}_{12} \cdot \mathrm{x}_{2 j}\right|^{2}}<\frac{\left(\left|\mathrm{x}_{2 j}^{2}\right|^{\frac{1}{2}}-\left|\mathrm{x}_{12}^{2}\right|^{\frac{1}{2}}\right)^{2}}{4} .
\end{aligned}
$$

Outside the domain of convergence (3.16), the correlations of $V_{1}\left(\mathrm{x}_{1}, \mathrm{x}_{2}\right)$ have to be defined by analytic continuation. When the correlations are rational, $V_{1}$ is Huygens bilocal, but the counter-example presented in Sect. 3.5 shows that rationality is not automatic. Then, it is not even obvious that the continuations are single-valued within the tube of analyticity required by the spectrum condition, i.e., that $V_{1}$ exists as a distribution in all of $M \times M$. Nontrivial case studies, however, show that at least for $\mathrm{x}_{k}$ space-like to both $\mathrm{x}_{1}$ and $\mathrm{x}_{2}$, the continuation is single-valued and preserves the independence on the position $k$ in (3.10) where $V_{1}\left(\mathrm{x}_{1}, \mathrm{x}_{2}\right)$ is inserted. This leads us to conjecture

Conjecture 3.3. The twist two field $V_{1}\left(\mathrm{x}_{1}, \mathrm{x}_{2}\right)$, whose correlations are defined as the analytic continuations of the harmonic parts of those of $U\left(\mathrm{x}_{1}, \mathrm{x}_{2}\right)$, exists and is bilocal in the ordinary sense, i.e., it commutes with $\phi(\mathrm{x})$ and $V_{1}\left(\mathrm{x}, \mathrm{x}^{\prime}\right)$ if $\mathrm{x}$ and $\mathrm{x}^{\prime}$ are space-like to $\mathrm{x}_{1}$ and $\mathrm{x}_{2}$.

We hope to return to this conjecture elsewhere (see also the Note added in proof). Note that the argument that locality implies Huygens locality [14] does not pass to bilocal fields.

3.2. Consequences of bi-harmonicity. Now our objective is to find the harmonic decomposition of the rational functions $F\left(\mathrm{x}_{1}, \mathrm{x}_{2}\right)$ that depend on $\mathrm{x}_{1}$ and $\mathrm{x}_{2}$ through the intervals $\rho_{i k}=\left(\mathrm{x}_{i}-\mathrm{x}_{k}\right)^{2}, i=1,2, k=3, \ldots, n$, for some additional points $\mathrm{x}_{3}, \ldots, \mathrm{x}_{n}$. The $F$ 's, as correlation functions of $U\left(\mathrm{x}_{1}, \mathrm{x}_{2}\right)$, have the form

$$
\begin{aligned}
F\left(\mathrm{x}_{1}, \mathrm{x}_{2}\right) & =\sum_{q=0}^{M}\left(\rho_{12}\right)^{q} F_{q}\left(\mathrm{x}_{1}, \mathrm{x}_{2}\right) \equiv \sum_{q=0}^{M}\left(\rho_{12}\right)^{q} F_{q}\left(\left\{\rho_{i k}\right\}_{\{i, k\} \neq\{1,2\}}\right), \\
F_{q}\left(\mathrm{x}_{1}, \mathrm{x}_{2}\right) & =\sum_{\left\{\mu_{1 i}\right\},\left\{\mu_{2 i}\right\}} C_{q,\left\{\mu_{1 j}\right\},\left\{\mu_{2 j}\right\}} \prod_{j=3}^{n}\left(\rho_{1 j}\right)^{\mu_{1 j}} \prod_{j=3}^{n}\left(\rho_{2 i}\right)^{\mu_{2 j}},
\end{aligned}
$$

where $M \in \mathbb{N}$ and $\mu_{1 j}, \mu_{2 j}(j=3, \ldots, n)$ are integers $>-d$ such that $\sum_{j \geqslant 3} \mu_{1 j}=$ $\sum_{j \geqslant 3} \mu_{2 j}=-1-q$, and the coefficients $C_{q,\left\{\mu_{1 j}\right\},\left\{\mu_{2 j}\right\}}$ may depend on $\rho_{j k}(j, k \geqslant 3)$.

If $H$ is the harmonic part of $F$ in $\mathrm{x}_{12}$, then the leading part $F_{0}$ (of order $\left.\left(\rho_{12}\right)^{0}\right)$ is also the leading part of $H$. We shall now proceed to show that bi-harmonicity of $H$ (Theorem 2.1), together with the first principles of QFT including GCI, implies strong constraints on $F_{0}$. 
Proposition 3.4. Let $F_{0}\left(\mathrm{x}_{1}, \mathrm{x}_{2}\right)$ be as in (3.18), and let $H\left(\mathrm{x}_{1}, \mathrm{x}_{2}\right)$ be its harmonic part with respect to $\mathrm{x}_{1}$ around $\mathrm{x}_{2}$. Then $H$ is also harmonic with respect to $\mathrm{x}_{2}$, if and only if $F_{0}$ satisfies the differential equation

$$
\left(E_{1} D_{2}-E_{2} D_{1}\right) F_{0}=0
$$

where $E_{1}=\sum_{i=3}^{n} \rho_{2 i} \partial_{1 i}$ (with $\partial_{j k}=\partial_{k j}=\frac{\partial}{\partial \rho_{j k}}$ ), $D_{1}=\sum_{3 \leqslant j<k \leqslant n} \rho_{j k} \partial_{1 j} \partial_{1 k}$, and similarly for $E_{2}$ and $D_{2}$, exchanging $1 \leftrightarrow 2$.

Proof. By Proposition 3.2 (see also Remark 3.1) we can consider $H$ as a function in the $2 n-3$ variables $\rho_{1 i}, \rho_{2 i}(i \geqslant 3)$ and $\rho_{12}$, analytic in some domain that includes $\rho_{12}=0$.

Expanding $H=\sum_{q}\left(\rho_{12}\right)^{q} H_{q} / q$ !, the functions $H_{q}$ are homogeneous of degree $-1-q$ in both sets of variables $\rho_{1 i}$ and $\rho_{2 i}$, and $H_{0}=F_{0}$. To impose the harmonicity with respect to the variable $\mathrm{x}_{1}$, we use the identity [11, App. C]

$$
\square_{\mathrm{x}_{1}} F=-\left.4\left[\sum_{2 \leqslant i<j \leqslant n} \rho_{i j} \partial_{1 i} \partial_{1 j} F\right]\right|_{\rho_{i j}=\left(\mathrm{x}_{i}-\mathrm{x}_{j}\right)^{2}},
$$

valid for homogeneous functions of $\rho_{1 i}$ of degree -1 , to express the wave operator $\square_{\mathrm{x}_{1}}$ as a differential operator with respect to the set of variables $\rho_{1 i}(i \geqslant 2)$. This yields the recursive system of differential equations

$$
E_{1} H_{q+1}=-D_{1} H_{q}
$$

Performing the same steps with respect to the variable $\mathrm{x}_{2}$, one obtains

$$
E_{2} H_{q+1}=-D_{2} H_{q} .
$$

Equation (3.19) then arises as the integrability condition for the pair of inhomogeneous differential equations for $H_{1}$ (putting $q=0$ ), observing that $E_{2} E_{1}-E_{1} E_{2}=$ $\sum \rho_{1 i} \partial_{1 i}-\sum \rho_{2 i} \partial_{2 i}$ vanishes on $H_{1}$ by homogeneity.

Conversely, if (3.19) is fulfilled, then $H_{1}$ exists and satisfies $\left(D_{1} E_{2}-D_{2} E_{1}\right) H_{1}=$ $-\left(D_{1} D_{2}-D_{2} D_{1}\right) H_{0}=0$ because $D_{1}$ and $D_{2}$ commute. But this is equivalent to $\left(D_{2} E_{1}-D_{1} E_{2}\right) H_{1}=0$, which is in turn the integrability condition for the existence of $\mathrm{H}_{2}$, and so on. It follows that bi-harmonicity imposes no further conditions on the leading function $H_{0}=F_{0}$.

The differential equation (3.19) imposes the following constraints on the leading part $F_{0}$ of the rational correlation function $F$ (3.17):

Corollary 3.5. Assume that the function $F_{0}$ as in (3.18) satisfies the differential equation (3.19). Then

(i) If $F_{0}$ contains a "double pole" of the form $\left(\rho_{1 i}\right)^{\mu_{1 i}}\left(\rho_{1 j}\right)^{\mu_{1 j}}$ with $i \neq j$ and $\mu_{1 i}$ and $\mu_{1 j}$ both negative, then its coefficients must be regular in $\rho_{2 k}(k \neq i, j)$.

(ii) $F_{0}$ cannot contain a "triple pole" of the form $\left(\rho_{1 i}\right)^{\mu_{1 i}}\left(\rho_{1 j}\right)^{\mu_{1 j}}\left(\rho_{1 k}\right)^{\mu_{1 k}}$ with $i, j, k$ all different and $\mu_{1 i}, \mu_{1 j}, \mu_{1 k}$ all negative.

The same hold true, exchanging $1 \leftrightarrow 2$. 
Proof. Pick any variable, say $\rho_{2 k}$, and decompose $F_{0}=\sum_{r \geqslant-p}\left(\rho_{2 k}\right)^{r} f_{r}$ as a Laurent polynomial in $\rho_{2 k}$. The differential equation (3.19) turns into the recursive system

$$
\left(\rho_{1 k} \sum_{i<j} \rho_{i j} \partial_{1 i} \partial_{1 j}-\sum_{i, j \neq k} \rho_{2 i} \rho_{k j} \partial_{1 i} \partial_{2 j}\right) r \cdot f_{r}=X_{r} f_{r-1}+Y f_{r}
$$

of differential equations for the functions $f_{r}$ which are Laurent polynomials in the remaining variables. The precise form of the polynomial differential operators $X_{r}$ and $Y$ does not matter. Assume the lowest power $-p$ of $\rho_{2 k}$ to be negative. For $r=-p$, the right-hand-side vanishes. Because the term $\rho_{i j} \partial_{1 i} \partial_{1 j}$ on the left-hand-side would produce a singularity that cannot be cancelled by any other term, $f_{-p}$ cannot have a "double pole" in any pair of variables $\rho_{1 i}, \rho_{1 j}$ with $i \neq j$ and $i, j \neq k$. This property passes recursively to all $f_{r}$ with $r<0$, because also the right-hand-side never can contain such a pole. This implies that a double pole in a pair of variables $\rho_{1 i}, \rho_{1 j}$ with $i \neq j$ cannot multiply a term that is singular in $\rho_{2 k}$ unless $k=i$ or $k=j$, proving (i).

If the coefficient of the double pole were singular in $\rho_{1 k}, k \neq i, j$, then the resulting double pole in the pair $\rho_{1 i}, \rho_{1 k}$ resp. $\rho_{1 j}, \rho_{1 k}$ would imply regularity also in $\rho_{2 j}$ resp. $\rho_{2 i}$. Hence the coefficient of a triple pole must be regular in all $\rho_{2 m}$, which contradicts the total homogeneity -1 of $F_{0}$ in these variables. This proves the statement (ii).

\subsection{A necessary and sufficient condition for Huygens bilocality.}

Definition 3.1. ("Single Pole Property", SPP). Let $f\left(\mathrm{x}_{1}, \ldots, \mathrm{x}_{n}\right)$ be a Laurent polynomial in the variables $\rho_{i j}$, i.e., regarded as a function of $\mathrm{x}_{1}$ only, it is a finite linear combination of functions of the form

$$
\prod_{j \geqslant 2}\left(\rho_{1 j}\right)^{\mu_{1 j}} \equiv \prod_{j \geqslant 2}\left[\left(\mathrm{x}_{1}-\mathrm{x}_{j}\right)^{2}\right]^{\mu_{1 j}},
$$

where $\mu_{1 j}(j \geqslant 2)$ are integers and the coefficients may depend on the parameters $\rho_{j k}$ $(j, k \geqslant 2)$. Then $f$ is said to satisfy the single pole property with respect to $\mathrm{x}_{1}$ if it contains no terms for which there are $j \neq k(j, k \geqslant 2)$ such that both $\mu_{1, j}$ and $\mu_{1, k}$ are negative.

The significance of SPP stems from the fact that the harmonic parts $H$ of $F_{0}$, i.e., the correlation functions of $V_{1}$, are again Laurent polynomials if and only if $F_{0}$ satisfies the SPP. Namely, if $H$ is a harmonic Laurent polynomial, the same argument as in [11, Lemma C.1] (using the representation (3.20) of the wave operator) shows that $H$ fulfills the SPP with respect to $\mathrm{x}_{1}$, and so does $F_{0}$, because it is the leading part of order $\left(\rho_{12}\right)^{0}$ of $H$. The converse is an immediate consequence of Lemma 3.6 (allowing for a relabelling and multiple counting of the points $\mathrm{x}_{3}, \ldots, \mathrm{x}_{n}$, which are not required to be distinct).

Lemma 3.6. Let $n \geqslant 4$. Every finite linear combination of monomials of the form

$$
g_{n}\left(\mathrm{x}_{1}\right)=\frac{\prod_{i=4}^{n} \rho_{1 i}}{\left(\rho_{13}\right)^{n-2}} \equiv \frac{\prod_{i=4}^{n}\left(\mathrm{x}_{1}-\mathrm{x}_{i}\right)^{2}}{\left[\left(\mathrm{x}_{1}-\mathrm{x}_{3}\right)^{2}\right]^{n-2}}
$$

has a rational harmonic decomposition in $\mathrm{x}_{1}$ around $\mathrm{x}_{2}$,

$$
g_{n}\left(\mathrm{x}_{1}\right)=h_{n}\left(\mathrm{x}_{1}\right)+\left(\mathrm{x}_{1}-\mathrm{x}_{2}\right)^{2} \cdot \tilde{g}_{n}\left(\mathrm{x}_{1}\right),
$$


i.e., $h_{n}$ is harmonic with respect to $\mathrm{x}_{1}$ and $\tilde{g}_{n}$ is regular at $\mathrm{x}_{1}=\mathrm{x}_{2}$, and both $h_{n}$ and $\tilde{g}_{n}$ are rational. More precisely, $\left(\rho_{13}\right)^{n-2}\left(\rho_{23}\right)^{n-3} h_{n}$ is a homogeneous polynomial of total degree $2(n-3)$ in the variables $\left\{\rho_{i j}: 1 \leqslant i<j\right\}$, which is separately homogenous of degree $n-3$ in the variables $\left\{\rho_{1 i}: i \geqslant 2\right\}$ and in the variables $\left\{\rho_{12}, \rho_{2 i}: i \geqslant 3\right\}$.

Proof. It is convenient to introduce the variables

$$
t_{i}=\frac{\rho_{1 i} \rho_{23}}{\rho_{13} \rho_{2 i}}, \quad s_{i}=\frac{\rho_{12} \rho_{3 i}}{\rho_{13} \rho_{2 i}}, \quad u_{i j}=\frac{\rho_{12} \rho_{23} \rho_{i j}}{\rho_{13} \rho_{2 i} \rho_{2 j}} \quad(4 \leqslant i<j \leqslant n) .
$$

We claim that $h_{n}\left(\mathrm{x}_{1}\right)$ is of the form

$$
h_{n}\left(\mathrm{x}_{1}\right)=\left(\prod_{i=4}^{n} \frac{\rho_{2 i}}{\rho_{23}}\right) \cdot \frac{f_{n}\left(t_{i}, s_{i}, u_{i j}\right)}{\rho_{13}},
$$

where $f_{n}$ are polynomials of degree $n-3$ such that $f_{n}\left(t_{i}, s_{i}=0, u_{i j}=0\right)=\prod_{i=4}^{n} t_{i}$. Because all $s_{i}$ and $u_{i j}$ contain a factor $\rho_{12}$, these properties ensure that $\tilde{g}_{n}$ given by $\left(g_{n}-h_{n}\right) / \rho_{12}$ is regular in $\rho_{12}$.

Using again the identity (3.20) for the wave operator, and transforming this into a differential operator with respect to the set of variables (3.26), we find

$$
\square_{\mathrm{x}_{1}} h_{n}\left(\mathrm{x}_{1}\right)=-4\left(\prod_{i=4}^{n} \frac{\rho_{2 i}}{\rho_{23}}\right) \frac{\rho_{23}}{\left(\rho_{13}\right)^{2} \rho_{12}} \cdot D f_{n}\left(t_{i}, s_{i}, u_{i j}\right),
$$

where $D$ is the differential operator

$$
D=\left(1+t \partial_{t}+s \partial_{s}+u \partial_{u}\right)\left(s \partial_{t}+s \partial_{s}+u \partial_{u}\right)-\left(s \partial_{s}+u \partial_{u}\right) \partial_{t}-u \partial_{t} \partial_{t}
$$

with shorthand notations for degree-preserving operators

$$
t \partial_{t}=\sum_{i=4}^{n} t_{i} \partial_{t_{i}}, \quad s \partial_{t}=\sum_{i=4}^{n} s_{i} \partial_{t_{i}}, \quad s \partial_{s}=\sum_{i=4}^{n} s_{i} \partial_{s_{i}}, \quad u \partial_{u}=\sum_{4 \leqslant i<j \leqslant n} u_{i j} \partial_{u_{i j}}
$$

and degree-lowering operators

$$
\partial_{t}=\sum_{i=4}^{n} \partial_{t_{i}}, \quad u \partial_{t} \partial_{t}=\sum_{4 \leqslant i<j \leqslant n} u_{i j} \partial_{t_{i}} \partial_{t_{j}}
$$

To solve the condition $D f_{n}=0$ for harmonicity, we make an ansatz

$$
f_{n}\left(t_{i}, s_{i}, u_{i j}\right)=\sum_{K \subset N} g_{K}^{(n)}\left(s_{k}, u_{k l}\right) \cdot \prod_{i \in N \backslash K}\left(t_{i}-s_{i}\right),
$$

where $N \equiv\{4, \ldots, n\}, g_{K}^{(n)}$ are polynomials in the variables $s_{k}, u_{k l}(k, l \in K)$ only, and $g_{\emptyset}^{(n)}=1$. Then the harmonicity condition $D f_{n}=0$ is equivalent to the recursive system

$$
(n-2-|K|+\Delta) \Delta g_{K}^{(n)}=\Delta \sum_{k \in K} g_{K \backslash\{k\}}^{(n)}+\sum_{k, l \in K, k<l}\left(u_{k l}-s_{k}-s_{l}\right) g_{K \backslash\{k, l\}}^{(n)},
$$

where $|K|$ is the number of elements of the set $\mathrm{K}$ and the differential operator $\Delta=$ $s \partial_{s}+u \partial_{u}$ measures the total polynomial degree $r$ in $s_{k}$ and $u_{k l}$. Since one can divide by 
$(n-2-|K|+r) r$ if $r>0$, there is a unique polynomial solution such that $g_{K}^{(n)}\left(s_{k}=\right.$ $\left.0, u_{k l}=0\right)=0(K \neq \emptyset)$, and $g_{K}^{(n)}$ is of order $\leqslant|K|$. So $f_{n}$ is of order $n-3$. (Explicitly, the first three functions are $f_{3}=1, f_{4}=t_{4}-s_{4}$ and $f_{5}=\left(t_{4}-s_{4}\right)\left(t_{5}-s_{5}\right)+\frac{1}{2}\left(u_{45}-\right.$ $\left.s_{4}-s_{5}\right)$.) An inspection of the recursion also shows that all possible factors $\rho_{2 i}$ in the denominators of the arguments of $f_{n}$ cancel with the factors in the prefactor in (3.27), thus $h_{n}$ can have poles only in $\rho_{13}$ and $\rho_{23}$ of the specified maximal degree. This proves the lemma.

The upshot of the previous discussion is a necessary and sufficient condition for the Huygens bilocality of $V_{1}$ which directly refers to the local correlation functions of the theory:

Theorem 3.7. The field $V_{1}\left(\mathrm{x}_{1}, \mathrm{x}_{2}\right)$ weakly converges on bounded energy states to a Huygens bilocal field which is conformal of weight $(1,1)$, if and only if the leading parts $F_{0}$ of the Laurent polynomials $F$ (3.17) satisfy the "single pole property" (Def. 3.1) with respect to both $\mathrm{x}_{1}$ and $\mathrm{x}_{2}$. In this case, the formal series $H$ converge to Laurent polynomials in $\left(\mathrm{x}_{i}-\mathrm{x}_{j}\right)^{2}$ subject to the same pole bounds, specified in Theorem 2.1, as $F$.

Proof. We know already that if $V_{1}$ is a Huygens bilocal field, then its correlation functions $H$ are Laurent polynomials of the form (2.3), and that this implies the SPP for $F_{0}$ with respect to $\mathrm{x}_{1}$ and $\mathrm{x}_{2}$. Conversely, if the SPP holds for $F_{0}$ with respect to $\mathrm{x}_{1}$ and $\mathrm{x}_{2}$, then $H$ are Laurent polynomials by Lemma 3.6, and hence $V_{1}$ is relatively Huygens bilocal with respect to the fields $\phi_{i}$. Since the general argument [4] that relative locality implies local commutativity of a field with itself refers only to local fields, we want to give an explicit argument for the case at hand.

All the previous remains true when in (3.10) or (3.17) a product of fields $\phi_{k}\left(\mathrm{x}_{k}\right)_{k+1} \phi\left(\mathrm{x}_{k+1}\right)$ is replaced by $U\left(\mathrm{x}_{k}, \mathrm{x}_{k+1}\right)$. By assumption, and because $U$ is bilocal, the contributions of order $\left(\rho_{k, k+1}\right)^{0}$ to the correlation functions of $U\left(\mathrm{x}_{k}, \mathrm{x}_{k+1}\right)$ fulfill the SPP with respect to $\mathrm{x}_{k}$ and $\mathrm{x}_{k+1}$. By Lemma 3.6, this property is preserved upon the passage to the harmonic parts with respect to $\mathrm{x}_{1}$ and $\mathrm{x}_{2}$. One may therefore continue in the same way with $\mathrm{x}_{k}, \mathrm{x}_{k+1}$, and eventually find that all mixed correlation functions of $\phi$ 's and $V_{1}$ 's converge to rational functions. By this convergence we conclude that all products of $\phi$ 's and $V_{1}$ 's converge on the vacuum, and this then defines $V_{1}$ as a Huygens bilocal field, since its matrix elements will satisfy Huygens locality.

The conformal properties of $V_{1}$ follow from the preservation of the homogeneity and the pole degrees in the harmonic decomposition, as guaranteed by Lemma 3.6.

For $n=4$ points, the SPP is trivially satisfied because of homogeneity. Hence the 4-point function $\left\langle 0\left|V_{1}^{*} V_{1}\right| 0\right\rangle$ is always rational. It follows that its expansion in (transcendental) partial waves [11] cannot terminate. This means that (unless $V_{1}=0$ in which case there is not even a stress-energy tensor) a GCI QFT necessarily contains infinitely many conserved tensor fields of arbitrarily high spin.

3.4. The case of dimension 2. Let us consider now the case of scalar fields $\phi_{k}$ of dimension 2. We claim that in this case, Corollary 3.5 in combination with the cluster condition is sufficient to establish the SPP, Definition 3.1. Hence we conclude by Theorem 3.7 that the twist two harmonic fields $V_{1}\left(\mathrm{x}_{1}, \mathrm{x}_{2}\right)$ are indeed Huygens bilocal fields.

To prove our claim, we use that by (2.6), $\mu_{i j} \geqslant-1$, hence the SPP is equivalent to the statement that there can be no term contributing to $\left\langle 0\left|\phi_{1}\left(\mathrm{x}_{1}\right) \cdots \phi_{n}\left(\mathrm{x}_{n}\right)\right| 0\right\rangle$, for which 
there is $i$ with more than two $\mu_{i j}$ negative $(j \neq i)$. Thus assume that there is a term with, say, $\mu_{12}=\mu_{13}=\mu_{14}=-1$. It constitutes a double pole for each of the three harmonic fields $V_{1}\left(\mathrm{x}_{1}, \mathrm{x}_{j}\right)(j=2,3,4)$. Then by homogeneity (2.5), there must be more poles in $\mathrm{x}_{j}(j=2,3,4)$, but these cannot be of the form $\rho_{j k}$ with $k>4$ by Corollary 3.5. Hence (up to permutations of $2,3,4) \mu_{23}=\mu_{24}=-1, \mu_{34}=0$. Again by homogeneity (2.5), the dependence on $\mathrm{x}_{1}, \ldots, \mathrm{x}_{4}$ must be given by a linear combination of terms

$$
\frac{\rho_{1 k} \rho_{4 \ell}}{\rho_{12} \rho_{13} \rho_{14} \rho_{23} \rho_{24}}
$$

with $k, \ell>4$. Applying the cluster limit (Sect. 2.1) to the points $\mathrm{x}_{1}, \mathrm{x}_{2}, \mathrm{x}_{3}, \mathrm{x}_{4}$ in (3.30), the limit diverges $\sim t^{4}$. This behavior is tamed to $\sim t^{2}$ by anti-symmetrization in $k, \ell$, but it cannot be cancelled by any other terms. Hence the assumption leads to a contradiction.

This proves the SPP if the generating scalar fields have dimension $d=2$.

3.5. A $d=4$ 6-point function violating the SPP. We proceed with an example of a 6-point function violating the SPP in the case of two $d=4$ GCI scalar fields $L_{i}(\mathrm{x})$ such that the bilocal field $U\left(\mathrm{x}_{1}, \mathrm{x}_{2}\right)$ obtained from $L_{1}\left(\mathrm{x}_{1}\right) L_{2}\left(\mathrm{x}_{2}\right)$ has a non-zero skew-symmetric part. Let $L$ be any linear combination of $L_{1}$ and $L_{2}$.

The following admissible contribution to the truncated part of the 6-point function $\left\langle 0\left|U\left(\mathrm{x}_{1}, \mathrm{x}_{2}\right) L\left(\mathrm{x}_{3}\right) L\left(\mathrm{x}_{4}\right) U\left(\mathrm{x}_{5}, \mathrm{x}_{6}\right)\right| 0\right\rangle$ clearly violates the SPP:

$$
F_{0}\left(\mathrm{x}_{1}, \mathrm{x}_{2}\right)=\mathbb{A}_{12} \mathbb{A}_{56}\left[\frac{\rho_{15} \rho_{26} \rho_{34}-2 \rho_{15} \rho_{23} \rho_{46}-2 \rho_{15} \rho_{24} \rho_{36}}{\rho_{13} \rho_{14} \rho_{23} \rho_{24} \cdot \rho_{34} \cdot \rho_{35} \rho_{45} \rho_{36} \rho_{46}}\right],
$$

where $\mathbb{A}_{i j}$ stands for the antisymmetrization in the arguments $\mathrm{x}_{i}, \mathrm{x}_{j}$. It is admissible as a truncated 6-point structure because $\left(\rho_{12} \rho_{56}\right)^{-3} F_{0}$ obeys all the pole bounds of Sect. 2 for a correlation $\left\langle 0\left|L_{1}\left(\mathrm{x}_{1}\right) L_{2}\left(\mathrm{x}_{2}\right) L\left(\mathrm{x}_{3}\right) L\left(\mathrm{x}_{4}\right) L_{1}\left(\mathrm{x}_{5}\right) L_{2}\left(\mathrm{x}_{6}\right)\right| 0\right\rangle^{\mathrm{tr}}$ of six fields of dimension $d=4$.

On the other hand, $F_{0}$ satisfies the differential equation

$$
\left(E_{1} D_{2}-E_{2} D_{1}\right) F_{0}\left(\mathrm{x}_{1}, \mathrm{x}_{2}\right)=0
$$

(and similar in the variables $\mathrm{x}_{5}$ and $\mathrm{x}_{6}$ ), ensuring that $F_{0}$ is the leading part of a bi-harmonic function, analytic in a neighborhood of $\mathrm{x}_{1}=\mathrm{x}_{2}$ and $\mathrm{x}_{5}=\mathrm{x}_{6}$, representing a contribution to the twist two 6-point function $\left\langle 0\left|V_{1}\left(\mathrm{x}_{1}, \mathrm{x}_{2}\right) L\left(\mathrm{x}_{3}\right) L\left(\mathrm{x}_{4}\right) V_{1}\left(\mathrm{x}_{5}, \mathrm{x}_{6}\right)\right| 0\right\rangle$, of which $F_{0}$ is the leading part. This function cannot be a Laurent polynomial in the $\rho_{i j}$ by our general argument that the leading part of a bi-harmonic Laurent polynomial cannot satisfy the SPP. Hence the twist two field $V_{1}\left(\mathrm{x}_{1}, \mathrm{x}_{2}\right)$ cannot be Huygens bilocal.

The resulting contribution to the conserved local current 4-point function $\left\langle 0\left|J_{\mu}\left(\mathrm{x}_{1}\right) L\left(\mathrm{x}_{3}\right) L\left(\mathrm{x}_{4}\right) J_{v}\left(\mathrm{x}_{5}\right)\right| 0\right\rangle^{\mathrm{tr}}$ is obtained through $J_{\mu}(\mathrm{x})=\left.i\left(\partial_{\mathrm{x}^{\mu}}-\partial_{\mathrm{y}^{\mu}}\right) V_{1}(\mathrm{x}, \mathrm{y})\right|_{\mathrm{x}=\mathrm{y}}$. It also satisfies the pertinent pole bounds. This structure is rational as it should be, because only the leading part $F_{0}$ contributes. In fact, while the 6-point structure involving the harmonic field cannot be reproduced by free fields due to its double pole, the resulting 4-point structure does arise as one of the three independent connected structures contributing to 4-point functions involving two Dirac currents : $\bar{\psi}_{a} \gamma^{\mu} \psi_{b}$ : and two Yukawa scalars $\varphi: \bar{\psi}_{c} \psi_{d}:$ (allowing for internal flavours $a, b, \ldots$ ). 


\section{The Theory of GCI Scalar Fields of Scaling Dimension $d=2$}

The scaling dimension $d=2$ is the minimal dimension of a GCI scalar field for which one could expect the existence of nonfree models. It turns out however, that in this case the fields can be constructed as composite fields of free, or generalized free, fields. Namely, we will establish the following result.

Theorem 4.1. Let $\left\{\Phi_{m}(\mathrm{x})\right\}_{m=1}^{\infty}$ be a system of real GCI scalar fields of scaling dimension $d=2$. Then it can be realized by a system of generalized free fields $\left\{\psi_{m}(\mathrm{x})\right\}$ and a system of independent real massless free fields $\left\{\varphi_{m}(\mathrm{x})\right\}$, acting on a possibly larger Hilbert space, as follows:

$$
\Phi_{m}(\mathrm{x})=\sum_{j=1}^{\infty} \alpha_{m, j} \psi_{j}(\mathrm{x})+\frac{1}{2} \sum_{j, k=1}^{\infty} \beta_{m, j, k}: \varphi_{j}(\mathrm{x}) \varphi_{k}(\mathrm{x}):,
$$

where $\alpha_{m, j}$ and $\beta_{m, j, k}=\beta_{m, k, j}$ are real constants such that $\sum_{j=1}^{\infty} \alpha_{m, j}^{2}<\infty$ and $\sum_{j, k=1}^{\infty} \beta_{m, j, k}^{2}<\infty$. Here, we assume the normalizations $\left\langle 0\left|\varphi_{j}\left(\mathrm{x}_{1}\right) \varphi_{k}\left(\mathrm{x}_{2}\right)\right| 0\right\rangle=$ $\delta_{j k}\left(\rho_{12}\right)^{-1},\left\langle 0\left|\psi_{j}\left(\mathrm{x}_{1}\right) \psi_{k}\left(\mathrm{x}_{2}\right)\right| 0\right\rangle=\delta_{j k}\left(\rho_{12}\right)^{-2}$.

The proof of Theorem 4.1 is given at the end of Sect. 4.2. The main reason for this result is the fact that in the $d=2$ case the harmonic bilocal fields exist and furthermore, they are Lie fields. This was originally recognized in $[12,2]$ under the assumption that there is a unique field $\phi$ of dimension 2 . We are extending here the result to an arbitrary system of $d=2 \mathrm{GCI}$ scalar fields.

If we assume the existence of a stress-energy tensor as a Wightman field ${ }^{5}$, the generalized free fields must be absent in (4.1), and the number of free fields must be finite. In this case, the iterated OPE generates in particular the bilocal field $\frac{1}{2} \sum_{i}: \varphi_{i}(\mathrm{x}) \varphi_{i}(\mathrm{y}):$. As this field has no other positive-energy representation than those occurring in the Fock space [2], nontrivial possibilities for correlations between non-free fields and the fields (4.1) are strongly limited.

4.1. Structure of the correlation functions. We consider a GCI QFT generated by a set of hermitian (real) scalar fields. We denote by $\mathcal{F}$ the real vector space of all GCI real scalar fields of scaling dimension 2 in the theory. (Note that the space $\mathcal{F}$ may be larger than the linear span of the original system of $d=2$ fields of Theorem 4.1.) We shall find in this section the explicit form of the correlation functions of the fields from $\mathcal{F}$.

Theorem 4.2. Let $\phi_{1}(\mathrm{x}), \ldots, \phi_{n}(\mathrm{x}) \in \mathcal{F}$, then their truncated $n$-point functions have the form

$$
\left\langle 0\left|\phi_{1}\left(\mathrm{x}_{1}\right) \cdots \phi_{n}\left(\mathrm{x}_{n}\right)\right| 0\right\rangle^{\mathrm{tr}}=\frac{1}{2 n} \sum_{\sigma \in \mathcal{S}_{n}} c^{(n)}\left(\phi_{\sigma_{1}}, \ldots, \phi_{\sigma_{n}}\right)\left(\rho_{\sigma_{1} \sigma_{2}} \cdots \rho_{\sigma_{n} \sigma_{1}}\right)^{-1},
$$

where $c^{(n)}$ are multilinear functionals $c^{(n)}: \mathcal{F}^{\otimes n} \rightarrow \mathbb{R}$ with the inversion and cyclic symmetries $c^{(n)}\left(\phi_{1}, \ldots, \phi_{n}\right)=c^{(n)}\left(\phi_{n}, \ldots, \phi_{1}\right)=c^{(n)}\left(\phi_{n}, \phi_{1}, \ldots, \phi_{n-1}\right)$.

\footnotetext{
${ }^{5}$ A stress-energy tensor always exists as a quadratic form between states generated by the fields $\Phi_{m}$ from the vacuum [8].
} 
Before we prove the theorem, let us first illustrate it on the example of the free field realization (4.1). In this case one finds

$$
\begin{aligned}
c^{(2)}\left(\Phi_{m_{1}}, \Phi_{m_{2}}\right) & =\sum_{j=1}^{\infty} \alpha_{m_{1}, j} \alpha_{m_{2}, j}+\sum_{j, k=1}^{\infty} \beta_{m_{1}, j, k} \beta_{m_{2}, j, k} \\
& \equiv \sum_{j=1}^{\infty} \alpha_{m_{1}, j} \alpha_{m_{2}, j}+\operatorname{Tr} \beta_{m_{1}} \beta_{m_{2}}, \\
c^{(n)}\left(\Phi_{m_{1}}, \ldots, \Phi_{m_{n}}\right) & =\operatorname{Tr} \beta_{m_{1}} \cdots \beta_{m_{n}} \text { for } n>2,
\end{aligned}
$$

where $\beta_{m}=\left(\beta_{m, j, k}\right)_{j, k}$.

Proof of Theorem 4.2. We first recall the general form (2.3) of the truncated correlation function with pole bounds (2.6) that read in this case: $\mu_{j k}^{\mathrm{tr}} \geqslant-1$. The argument in Sect. 3.4 shows that the nonzero contributing terms in Eq. (2.3) have for every $j=$ $1, \ldots, n$ exactly two negative $\mu_{j k}^{\mathrm{tr}}$ or $\mu_{k j}^{\mathrm{tr}}$ for some $k=k_{1}, k_{2}$ different from $j$.

The nonzero terms are therefore products of "disjoint cyclic products of propagators" of the form $1 / \rho_{k_{1} k_{2}} \rho_{k_{2} k_{3}} \cdots \rho_{k_{r-1} k_{r}} \rho_{k_{r} k_{1}}$. But cycles of length $r<n$ are in conflict with the cluster condition (Sect. 2). We conclude that $\left\langle 0\left|\phi_{1}\left(\mathrm{x}_{1}\right) \cdots \phi_{n}\left(\mathrm{x}_{n}\right)\right| 0\right\rangle^{\mathrm{tr}}$ is a linear combination of terms like those in (4.2) with some coefficients $c_{\sigma}\left(\phi_{1}, \ldots, \phi_{n}\right)$ depending on the permutations $\sigma \in \mathcal{S}_{n}$ and on the fields $\phi_{j}$ (multilinearly). Locality, i.e. $\left\langle 0\left|\phi_{1}\left(\mathrm{x}_{1}\right) \cdots \phi_{n}\left(\mathrm{x}_{n}\right)\right| 0\right\rangle^{\mathrm{tr}}=\left\langle 0\left|\phi_{\sigma_{1}^{\prime}}\left(\mathrm{x}_{\sigma_{1}^{\prime}}\right) \cdots \phi_{\sigma_{n}^{\prime}}\left(\mathrm{x}_{\sigma_{n}^{\prime}}\right)\right| 0\right\rangle^{\mathrm{tr}}$, then implies $c_{\sigma^{\prime} \sigma}\left(\phi_{1}, \ldots, \phi_{n}\right)$ $=c_{\sigma}\left(\phi_{\sigma_{1}^{\prime}}, \ldots, \phi_{\sigma_{n}^{\prime}}\right)\left(\sigma, \sigma^{\prime} \in \mathcal{S}_{n}\right)$, so that $c_{\sigma}\left(\phi_{1}, \ldots, \phi_{n}\right)=c^{(n)}\left(\phi_{\sigma_{1}}, \ldots, \phi_{\sigma_{n}}\right)$ for some $c^{(n)}: \mathcal{F}^{\otimes n} \rightarrow \mathbb{R}$. The equalities $c^{(n)}\left(\phi_{1}, \ldots, \phi_{n}\right)=c^{(n)}\left(\phi_{n}, \ldots, \phi_{1}\right)=c^{(n)}\left(\phi_{n}, \phi_{1}, \ldots\right.$, $\left.\phi_{n-1}\right)$ are again due to locality.

As we already know by the general results of the previous section, the harmonic bilocal field exists in the case of fields of dimension $d=2$. Moreover, the knowledge of the correlation functions of the $d=2$ fields allows us to find the form of the correlation functions of the resulting bilocal fields. This yields an algebraic structure in the space of real (local and bilocal) scalar fields, which we proceed to display.

Let us introduce together with the space $\mathcal{F}$ of $d=2$ fields also the real vector space $\mathcal{V}$ of all real harmonic bilocal fields. We shall consider $\mathcal{F}$ and $\mathcal{V}$ as built starting from our original system of $d=2$ fields $\left\{\Phi_{m}\right\}$ of Theorem 4.1, by the following constructions:

(a) If $\phi_{1}(\mathrm{x}), \phi_{2}(\mathrm{x}) \in \mathcal{F}$ then introducing the bilocal $(1,1)$-field $U\left(\mathrm{x}_{1}, \mathrm{x}_{2}\right)=$ $\mathrm{x}_{12}^{2}\left[\phi_{1}\left(\mathrm{x}_{1}\right) \phi_{2}\left(\mathrm{x}_{2}\right)-\left\langle 0\left|\phi_{1}\left(\mathrm{x}_{1}\right) \phi_{2}\left(\mathrm{x}_{2}\right)\right| 0\right\rangle\right]$ in accord with Eq. (2.7), we consider its harmonic decomposition $U(\mathrm{x}, \mathrm{y})=V_{1}(\mathrm{x}, \mathrm{y})+(\mathrm{x}-\mathrm{y})^{2} \widetilde{U}(\mathrm{x}, \mathrm{y})$. We denote $V_{1}(\mathrm{x}, \mathrm{y})$ by $\phi_{1} * \phi_{2} ;$ this defines a bilinear map $\mathcal{F} \otimes \mathcal{F} \stackrel{*}{\rightarrow} \mathcal{V}$.

(b) If now $v(\mathrm{x}, \mathrm{y}) \in \mathcal{V}$ then $v^{t}(\mathrm{x}, \mathrm{y}):=v(\mathrm{y}, \mathrm{x})$ also belongs to $\mathcal{V}$ and $\gamma(v)(\mathrm{x})$ $:=\frac{1}{2} v(\mathrm{x}, \mathrm{x})$ is a field from $\mathcal{F}$.

(c) If $v(\mathrm{x}, \mathrm{y}), v^{\prime}(\mathrm{x}, \mathrm{y}) \in \mathcal{V}$ then there is a harmonic bilocal field

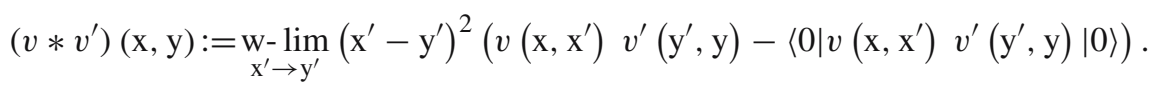

The existence of the above weak limit (i.e., a limit within correlation functions) will be established below together with the independence of $\mathrm{x}^{\prime}=\mathrm{y}^{\prime}$ and the regularity of the resulting field for $(x-y)^{2}=0$. 
(d) If $v(\mathrm{x}, \mathrm{y}) \in \mathcal{V}$ and $\phi(\mathrm{x}) \in \mathcal{F}$ then we can construct the following bilocal field belonging to $\mathcal{V}$ :

$$
(v * \phi)(\mathrm{x}, \mathrm{y}):=\underset{\mathrm{x}^{\prime} \rightarrow \mathrm{y}}{\mathrm{w}-\lim }\left(\mathrm{x}^{\prime}-\mathrm{y}\right)^{2}\left(v\left(\mathrm{x}, \mathrm{x}^{\prime}\right) \phi(\mathrm{y})-\left\langle 0\left|v\left(\mathrm{x}, \mathrm{x}^{\prime}\right) \phi(\mathrm{y})\right| 0\right\rangle\right),
$$

where again the existence of the limit and the regularity for $(x-y)^{2}=0$ will be established later.

One can define similarly a product $\phi * v \in \mathcal{V}$, but it would then be expressed as: $\left(v^{t} * \phi\right)^{t}$.

To summarize, we have three bilinear maps: $\mathcal{F} \otimes \mathcal{F} \stackrel{*}{\rightarrow} \mathcal{V}, \mathcal{V} \otimes \mathcal{V} \stackrel{*}{\rightarrow} \mathcal{V}, \mathcal{V} \otimes \mathcal{F} \stackrel{*}{\rightarrow} \mathcal{V}$, and two linear ones: $\mathcal{V} \stackrel{t}{\rightarrow} \mathcal{V}, \mathcal{V} \stackrel{\gamma}{\rightarrow} \mathcal{F}$. Applying these maps we construct $\mathcal{F}$ and $\mathcal{V}$ inductively, starting from our original system of $d=2$ fields, given in Theorem 4.1 , and at each step of this inductive procedure, we establish the existence of the above limits in (c) and (d). In fact, we shall establish this together with the structure of the truncated correlation functions for the fields in $\mathcal{F}$ and $\mathcal{V} .6$

Before we state the inductive result it is convenient to introduce the vector space

$$
\widehat{\mathcal{A}}=\mathcal{F} \times \mathcal{V}
$$

and endow it with the following bilinear operation:

$$
\left(\phi_{1}, v_{1}\right) *\left(\phi_{2}, v_{2}\right):=\left(0, \phi_{1} * \phi_{2}+v_{1} * v_{2}+v_{1} * \phi_{2}+\left(v_{2}^{t} * \phi_{1}\right)^{t}\right),
$$

and with the transposition

$$
(\phi, v)^{t}:=\left(\phi, v^{t}\right)
$$

The spaces $\mathcal{F}$ and $\mathcal{V}$ will be considered as subspaces in $\widehat{\mathcal{A}}$. Thus, the new operation $*$ in $\widehat{\mathcal{A}}$ combines the above listed three operations. We shall see later that $\widehat{\mathcal{A}}$ is actually an associative algebra under the product (4.7). We note that the transposition $t$ (4.8) is an antiinvolution with respect to the product: $\left(q_{1} * q_{2}\right)^{t}=q_{2}^{t} * q_{1}^{t}$, for every $q_{1}, q_{2} \in \widehat{\mathcal{A}}$.

Proposition 4.3. There exist multilinear functionals

$$
c^{(N)}: \widehat{\mathcal{A}}^{\otimes N} \rightarrow \mathbb{R}
$$

such that if we take elements $q_{1}, \ldots, q_{n+m} \in \widehat{\mathcal{A}}: q_{k}:=v_{k}\left(\mathrm{x}_{k[0]}, \mathrm{x}_{k[1]}\right) \in \mathcal{V}$, where $[\varepsilon]$ stands for $a \mathbb{Z} / 2 \mathbb{Z}$-value and $k=1, \ldots, n$, and $q_{k}:=\phi_{k-n}\left(\mathrm{x}_{k}\right) \in \mathcal{F}$ for $k=$ $n+1, \ldots, n+m$, then the truncated correlation functions can be written in the following form:

$$
\begin{aligned}
& \left\langle 0\left|v_{1}\left(\mathrm{x}_{1[0]}, \mathrm{x}_{1[1]}\right) \cdots v_{n}\left(\mathrm{x}_{n[0]}, \mathrm{x}_{n[1]}\right) \phi_{1}\left(\mathrm{x}_{n+1}\right) \cdots \phi_{m}\left(\mathrm{x}_{n+m}\right)\right| 0\right\rangle^{\mathrm{tr}} \\
& =\frac{1}{2(n+m)} \sum_{\substack{\sigma \in \mathcal{S}_{n+m} \\
\left(\varepsilon_{1}, \ldots, \varepsilon_{n}\right) \in(\mathbb{Z} / 2 \mathbb{Z})^{n}}} K_{\sigma, \varepsilon} T_{\sigma, \varepsilon}\left(\mathrm{x}_{1[0]}, \ldots, \mathrm{x}_{n[1]}, \mathrm{x}_{n+1}, \ldots, \mathrm{x}_{n+m}\right)^{-1} .
\end{aligned}
$$

6 Since we shall use the notion of truncated correlation functions also for bilocal fields, let us briefly recall it. If $B_{1}, \ldots, B_{n}$ are some smeared (multi)local fields then their truncated correlation functions are recursively defined by: $\left\langle 0\left|B_{1} \cdots B_{n}\right| 0\right\rangle=\sum_{\dot{U} P=\{1, \ldots, n\}} \prod_{\left\{j_{1}, \ldots, j_{k}\right\} \in P}\left\langle 0\left|B_{j_{1}} \cdots B_{j_{k}}\right| 0\right\rangle^{\mathrm{tr}}$ (the sum being over all partitions $P$ of $\{1, \ldots, n\})$. 
Here: $K_{\sigma, \varepsilon}$ are coefficients given by $K_{\sigma, \varepsilon}:=c^{(n+m)}\left(q_{\sigma_{1}}^{\left[\varepsilon_{\sigma_{1}}\right]}, \ldots, q_{\sigma_{n+m}}^{\left[\varepsilon_{\sigma_{n+m}}\right]}\right)$, where we set $\varepsilon_{n+1}=\cdots=\varepsilon_{n+m}=0$, and $q^{[0]}:=q, q^{[1]}:=q^{t}($ for $q \in \widehat{\mathcal{A}}) ;$ the terms $T_{\sigma, \varepsilon}$ are the following cyclic products of intervals:

$$
\begin{aligned}
T_{\sigma, \varepsilon}= & \left(\mathrm{x}_{\sigma_{n+m}}-\mathrm{x}_{\sigma_{1}\left[\varepsilon_{1}\right]}\right)^{2} \prod_{k=1}^{n-1}\left(\mathrm{x}_{\sigma_{k}\left[1+\varepsilon_{k}\right]}-\mathrm{x}_{\sigma_{k+1}\left[\varepsilon_{k+1}\right]}\right)^{2} \\
& \times\left(\mathrm{x}_{\sigma_{n}\left[1+\varepsilon_{n}\right]}-\mathrm{x}_{\sigma_{n+1}}\right)^{2} \prod_{k=1}^{m-1}\left(\mathrm{x}_{\sigma_{n+k}}-\mathrm{x}_{\sigma_{n+k+1}}\right)^{2}
\end{aligned}
$$

It follows by Eq. (4.10) that the limits in the steps (c) and (d) above are well defined.

Before the proof let us make some remarks. First, we used the same notation $c^{(n)}$ as in Theorem 4.2 since the above multilinear functionals are obviously an extension of the previous, i.e., Eq. (4.10) reduces to Eq. (4.2) for $m=0$. Let us also give an example for Eq. (4.10) with $n=m=1$ :

$$
\begin{aligned}
& \left\langle 0\left|v\left(\mathrm{x}_{1}, \mathrm{x}_{2}\right) \phi\left(\mathrm{x}_{3}\right)\right| 0\right\rangle=\frac{1}{4}\left(c^{(2)}(v, \phi)\left(\rho_{23} \rho_{31}\right)^{-1}+c^{(2)}\left(v^{t}, \phi\right)\left(\rho_{13} \rho_{32}\right)^{-1}\right. \\
& \left.+c^{(2)}(\phi, v)\left(\rho_{31} \rho_{23}\right)^{-1}+c^{(2)}\left(\phi, v^{t}\right)\left(\rho_{32} \rho_{13}\right)^{-1}\right) .
\end{aligned}
$$

As one can see, $c^{(n)}$ (as well as $c^{(n)}$ of Theorem 4.2) possess a cyclic and an inversion symmetry:

$$
c^{(n)}\left(q_{1}, \ldots, q_{n}\right)=c^{(n)}\left(q_{n}, q_{1} \ldots, q_{n-1}\right)=c^{(n)}\left(q_{n}^{t}, \ldots, q_{1}^{t}\right) .
$$

This is the reason for choosing the prefactors in Eqs. (4.2) and (4.10) (the inverse of the orders of the symmetry groups).

Proof of Proposition 4.3. According to our preliminary remarks it is enough to prove that Eq. (4.10) is consistent with the operations $\mathcal{F} \otimes \mathcal{F} \stackrel{*}{\rightarrow} \mathcal{V}, \mathcal{V} \otimes \mathcal{V} \stackrel{*}{\rightarrow} \mathcal{V}, \mathcal{V} \otimes \mathcal{F} \stackrel{*}{\rightarrow} \mathcal{V}$ and $\mathcal{V} \stackrel{\gamma}{\rightarrow} \mathcal{F}$.

Starting with $\mathcal{F} \otimes \mathcal{F} \stackrel{*}{\rightarrow} \mathcal{V}$ one should prove that any truncated correlation function $\left\langle\cdot \phi_{1}\left(\mathrm{x}_{1}\right) \phi_{2}\left(\mathrm{x}_{2}\right) \cdot\right\rangle^{\mathrm{tr}}$ given by Eq. (4.10) yields a harmonic decomposition: $\rho_{12}$ $\left\langle\cdot \phi_{1}\left(\mathrm{x}_{1}\right) \phi_{2}\left(\mathrm{x}_{2}\right) \cdot\right\rangle^{\mathrm{tr}}=\left\langle\cdot\left(\phi_{1} * \phi_{2}\right)\left(\mathrm{x}_{1}, \mathrm{x}_{2}\right) \cdot\right\rangle^{\mathrm{tr}}+\rho_{12} R\left(\mathrm{x}_{1}, \mathrm{x}_{2}\right)$, with a correlation function $\left\langle\cdot\left(\phi_{1} * \phi_{2}\right)\left(\mathrm{x}_{1}, \mathrm{x}_{2}\right) \cdot\right\rangle^{\mathrm{tr}}$ given by Eq. (4.10) and a rational function $R$ regular at $\rho_{12}=0$. This gives us relations of the type

$$
c^{(n+2)}\left(q_{1}, \ldots, \phi_{1}, \phi_{2}, \ldots, q_{n}\right)=c^{(n+1)}\left(q_{1}, \ldots, \phi_{1} * \phi_{2}, \ldots, q_{n}\right) .
$$

Next, having correlation functions of type $\left\langle\cdot v_{1}\left(\mathrm{x}_{1}, \mathrm{x}_{2}\right) v_{2}\left(\mathrm{x}_{3}, \mathrm{x}_{4}\right) \cdot\right\rangle^{\mathrm{tr}}$ or $\left\langle\cdot v\left(\mathrm{x}_{1}, \mathrm{x}_{2}\right) \phi\left(\mathrm{x}_{3}\right) \cdot\right\rangle^{\mathrm{tr}}$ of the form (4.10), one verifies that the limits (4.4) and (4.5) exist within these correlation functions, and they yield expressions for $\left\langle\cdot\left(v_{1} * v_{2}\right)\left(\mathrm{x}_{1}, \mathrm{x}_{4}\right) \cdot\right\rangle^{\mathrm{tr}}$ and $\left\langle\cdot(v * \phi)\left(\mathrm{x}_{1}, \mathrm{x}_{3}\right) \cdot\right\rangle^{\mathrm{tr}}$ consistent with (4.10). As a result we obtain again relations between the $c$ 's:

$$
\begin{aligned}
c^{(n+2)}\left(q_{1}, \ldots, v_{1}, v_{2}, \ldots, q_{n}\right) & =c^{(n+1)}\left(q_{1}, \ldots, v_{1} * v_{2}, \ldots, q_{n}\right), \\
c^{(n+2)}\left(q_{1}, \ldots, v, \phi, \ldots, q_{n}\right) & =c^{(n+1)}\left(q_{1}, \ldots, v * \phi, \ldots, q_{n}\right) .
\end{aligned}
$$


Finally, one verifies that setting $\mathrm{x}_{1}=\mathrm{x}_{2}$ in $\left\langle\cdot v\left(\mathrm{x}_{1}, \mathrm{x}_{2}\right) \cdot\right\rangle^{\mathrm{tr}}$ we obtain the correlation functions $\left\langle\cdot \gamma(v)\left(\mathrm{x}_{1}\right) \cdot\right\rangle^{\mathrm{tr}}$ with the relation

$$
c^{(n+1)}\left(q_{1}, \ldots,\left(v+v^{t}\right), \ldots, q_{n}\right)=2 c^{(n+1)}\left(q_{1}, \ldots, \gamma(v), \ldots, q_{n}\right) .
$$

This completes the proof of Proposition 4.3 as well as the proof that the products $\mathcal{V} \otimes$ $\mathcal{V} \stackrel{*}{\rightarrow} \mathcal{V}$ and $\mathcal{V} \otimes \mathcal{F} \stackrel{*}{\rightarrow} \mathcal{V}$ are well defined.

4.2. Associative algebra structure of the OPE. Note that Eqs. (4.14), (4.15) read (under (4.7))

$$
c^{(n)}\left(q_{1}, \ldots, q_{k}, q_{k+1}, \ldots, q_{n}\right)=c^{(n-1)}\left(q_{1}, \ldots, q_{k} * q_{k+1}, \ldots, q_{n}\right) .
$$

This implies that the bilinear operation $*$ on $\widehat{\mathcal{A}}$ is an associative product.

Indeed, consider the element $q:=\left(q_{1} * q_{2}\right) * q_{3}-q_{1} *\left(q_{2} * q_{3}\right)$ for $q_{1}, q_{2}, q_{3} \in \widehat{\mathcal{A}}$. By (4.7) $q$ is a bilocal field. Equation (4.17) implies that all $c$ 's in which $q$ enters vanish and hence, by Eq. (4.10) $q$ has zero correlation functions with all other fields, including itself. But then this (bilocal) field is zero by the Reeh-Schlieder theorem, since its action on the vacuum will be identically zero.

Thus, introducing the cartesian product $\widehat{\mathcal{A}}(4.6)$ was not only convenient for combining three types of bilinear operations in one but also as a compact expression for the associativity (Eqs. (4.14), (4.15)). However, $\widehat{\mathcal{A}}$ carries a redundant information due to the following relation:

$$
\left(-\gamma(v), \frac{1}{2}\left(v+v^{t}\right)\right) * q=0=q *\left(-\gamma(v), \frac{1}{2}\left(v+v^{t}\right)\right)
$$

for every $v \in \mathcal{V}$ and $q \in \widehat{\mathcal{A}}$. To prove (4.18) we point out first that it is equivalent to the identities $v * \phi=\gamma(v) * \phi$ and $v^{\prime} * v=v^{\prime} * \gamma(v)$ for $v=v^{t} \in \mathcal{V}$ and any $\phi \in \mathcal{F}$, $v^{\prime} \in \mathcal{V}$. These identities can be established again first for the $c$ 's, and then proceeding by using the Reeh-Schlieder theorem, as in the above proof of associativity.

Hence, the redundancy in $\widehat{\mathcal{A}}$ is because we can identify symmetric bilocal fields $v=v^{t} \in \mathcal{V}$ with their restrictions to the diagonal, $\gamma(v) \in \mathcal{F}$, and this is compatible with the product $*$. Let us point out that the restriction of the map $\gamma$ to the $t$-invariant subspace $\mathcal{V}_{s}:=\left\{v \in \mathcal{V}: v=v^{t}\right\}$ is an injection into $\mathcal{F}$. The latter follows from a simple analysis of the 4-point functions of $v$ and the Reeh-Schlieder theorem: if $v(\mathrm{x}, \mathrm{y})$ $=v(\mathrm{y}, \mathrm{x})$ and $\langle 0|v(\mathrm{x}, \mathrm{x}) v(\mathrm{y}, \mathrm{y})| 0\rangle=0$ then $\left\langle 0\left|v\left(\mathrm{x}, \mathrm{x}^{\prime}\right) v\left(\mathrm{y}, \mathrm{y}^{\prime}\right)\right| 0\right\rangle=0$. In this way we see that we can identify in $\widehat{\mathcal{A}}$ the symmetric harmonic bilocal fields $v=v^{t}$ with their restriction on the diagonal $\gamma(v) \in \mathcal{F}$.

Formally, the above considerations can be summarized in the following abstract way. Let us introduce the quotient

$$
\mathcal{A}:=\widehat{\mathcal{A}} /\left\{\left(-\gamma(v), \frac{1}{2}\left(v+v^{t}\right)\right): v \in \mathcal{V}\right\} .
$$

It is an associative algebra according to Eq. (4.18). The involution $t: \widehat{\mathcal{A}} \rightarrow \widehat{\mathcal{A}}$ can be transferred to an involution on the quotient (4.19) and we denote it by $t$ as well. The spaces $\mathcal{F}$ and $\mathcal{V}$ are mapped into $\mathcal{A}$ by the natural compositions $\mathcal{F} \rightarrow \widehat{\mathcal{A}} \rightarrow \mathcal{A}$ and $\mathcal{V} \rightarrow \widehat{\mathcal{A}} \rightarrow \mathcal{A}$. The injectivity of $\gamma$ on $\mathcal{V}_{s}$ implies that the maps $\mathcal{F} \rightarrow \mathcal{A}$ and $\mathcal{V} \rightarrow \mathcal{A}$ 
so defined are actually injections. Hence, we shall treat $\mathcal{F}$ and $\mathcal{V}$ also as subspaces of $\mathcal{A}$. Furthermore, $\mathcal{A}$ becomes a direct sum of vector spaces

$$
\begin{aligned}
& \mathcal{A}=\mathcal{F} \oplus \mathcal{V}_{a} \\
& \text { with } \quad\left\{q \in \mathcal{A}: q^{t}=q\right\}=\mathcal{F} \supseteq \mathcal{V}_{s}\left(:=\left\{v \in \mathcal{V}: v^{t}=v\right\}\right) \text {, } \\
& \left\{q \in \mathcal{A}: q^{t}=-q\right\}=\mathcal{V}_{a}:=\left\{v \in \mathcal{V}: v^{t}=-v\right\} \text {. }
\end{aligned}
$$

Hence, the $t$-symmetric elements of $\mathcal{A}$ are identified with the $d=2$ local fields, while the $t$-antisymmetric elements of $\mathcal{A}$, with the antisymmetric, harmonic bilocal $(1,1)$ fields. (Neither $\mathcal{F}$ nor $\mathcal{V}_{a}$ are subalgebras of $\mathcal{A}$.)

To summarize, the associative algebra $\mathcal{A}$ is obtained from $\widehat{\mathcal{A}}$ by identifying the space $\mathcal{V}_{s}$ of symmetric bilocal fields with its image $\gamma\left(\mathcal{V}_{s}\right) \subseteq \mathcal{F}$.

For simplicity we will denote the equivalence class in $\mathcal{A}$ of an element $q \in \widehat{\mathcal{A}}$ again by $q$. Also note that the $c$ 's can be transferred as well, to multilinear functionals on $\mathcal{A}$, since the kernel of the quotient (4.19) is contained in the kernel of each $c^{(n)}$ by (4.16). We shall use the same notation $c^{(n)}$ also for the multilinear functional $c^{(n)}$ on $\mathcal{A}$.

Example 4.1. Let us illustrate the above algebraic structures on the simplest example of a QFT generated by a pair of $d=2$ GCI fields $\Phi_{1}$ and $\Phi_{2}$ given by normal a pair of two mutually commuting free massless fields $\varphi_{j}: \Phi_{1}(\mathrm{x})=\frac{1}{2}\left(: \varphi_{1}^{2}(\mathrm{x}):-: \varphi_{2}^{2}(\mathrm{x}):\right)$ and $\Phi_{2}(\mathrm{x})=\varphi_{1}(\mathrm{x}) \varphi_{2}(\mathrm{x})$. Their OPE algebra involves a set of four independent harmonic bilocal fields $V_{j k}\left(\mathrm{x}_{1}, \mathrm{x}_{2}\right):=: \varphi_{j}\left(\mathrm{x}_{1}\right) \varphi_{k}\left(\mathrm{x}_{2}\right):(j, k=1,2)$, which satisfy $\left[V_{j k}\left(\mathrm{x}_{1}, \mathrm{x}_{2}\right)\right]^{*}$ $=V_{k j}\left(\mathrm{x}_{1}, \mathrm{x}_{2}\right)=V_{j k}\left(\mathrm{x}_{2}, \mathrm{x}_{1}\right)$. For instance, we have $\Phi_{1} * \Phi_{2}=V_{12}-V_{21}{ }^{7}$ Also note that $\Phi_{1}=\gamma\left(V_{1}\right)$ for $V_{1}\left(\mathrm{x}_{1}, \mathrm{x}_{2}\right)=: \varphi_{1}\left(\mathrm{x}_{1}\right) \varphi_{1}\left(\mathrm{x}_{2}\right):-: \varphi_{2}\left(\mathrm{x}_{1}\right) \varphi_{2}\left(\mathrm{x}_{2}\right):$, etc.

By the associativity and Eq. (4.17) we have

$$
c^{(n)}\left(q_{1}, \ldots, q_{n}\right)=c^{(2)}\left(q_{1} * \cdots * q_{n-1}, q_{n}\right)
$$

for $q_{1}, \ldots, q_{n} \in \mathcal{A}$. Let us consider now $c^{(2)}$ and define the following symmetric bilinear form on $\mathcal{A}$ :

$$
\left\langle q_{1}, q_{2}\right\rangle:=c^{(2)}\left(q_{1}^{t}, q_{2}\right)
$$

First note that $\mathcal{F}$ and $\mathcal{V}_{a}$ are orthogonal with respect to this bilinear form: this is due to the fact that there is no nonzero three point conformally invariant scalar function of weights $(2,1,1)$, which is antisymmetric in the second and third arguments. Next, we claim that (4.22) is strictly positive definite. This is a straightforward consequence of the Wightman positivity and the Reeh-Schlieder theorem (one should consider separately the positivity on $\mathcal{F}$ and $\mathcal{V}_{a}$ ). In particular, (4.22) is nondegenerate. By Eqs. (4.13) and (4.17) we have:

$$
\left\langle q_{1} * q_{2}, q_{3}\right\rangle=\left\langle q_{2}, q_{1}^{t} * q_{3}\right\rangle
$$

for all $q_{1}, q_{2}, q_{3} \in \mathcal{A}$.

Let us introduce now an additional splitting of $\mathcal{F}$. Denote by $\mathcal{F}_{0}$ the kernel of the product, i.e.,

$$
\mathcal{F}_{0}:=\{\psi \in \mathcal{F}: \psi * q=0 \forall q \in \mathcal{A}\} \equiv\{\psi \in \mathcal{F}: q * \psi=0 \forall q \in \mathcal{A}\}
$$

7 I.e., in the OPE $\Phi_{1}\left(\mathrm{x}_{1}\right) \Phi_{2}\left(\mathrm{x}_{2}\right)$ there appears the antisymmetric bilocal field $V_{12}\left(\mathrm{x}_{1}, \mathrm{x}_{2}\right)-V_{21}\left(\mathrm{x}_{1}, \mathrm{x}_{2}\right)$ that involves only odd rank conserved tensor currents in its expansion in local fields. 
(the second equality is due to the identity $\left.\phi * q=\left(q^{t} * \phi\right)^{t}\right)$. Let $\mathcal{F}_{1}$ be the orthogonal complement in $\mathcal{F}$ of $\mathcal{F}_{0}$ with respect to the scalar product (4.22):

$$
\mathcal{F}_{1}:=\left\{\phi \in \mathcal{F}:\langle\phi, \psi\rangle=0 \forall \psi \in \mathcal{F}_{0}\right\} .
$$

The meaning of fields belonging to $\mathcal{F}_{0}$ becomes immediately clear if we note that $c^{(n)}$ for $n \geqslant 3$ are zero if one of the arguments belongs to $\mathcal{F}_{0}$ (this is due to Eq. (4.21)). Hence, all their truncated functions higher than two point are zero, i.e., the fields belonging to $\mathcal{F}_{0}$ are generalized free $d=2$ fields. Furthermore, these fields commute with all other fields from $\mathcal{F}_{1}$ and $\mathcal{V}_{a} \equiv \mathcal{A}^{(1)}$ : this is because of the vanishing of $c^{(2)}(\psi, q)$ if $\psi \in \mathcal{F}_{0}$ and $q \in \mathcal{F}_{1} \oplus \mathcal{V}_{a}$, as well as of all $c^{(n+1)}\left(\psi, q_{1}, \ldots, q_{n}\right)$ for $n \geqslant 2$ if $\psi \in \mathcal{F}_{0}$ and $q_{1}, \ldots, q_{n} \in \mathcal{A}$ (by (4.21) and (4.24)).

Clearly, $\mathcal{F}_{1} \oplus \mathcal{V}_{a}$ is a subalgebra of $\mathcal{A}$ : this follows from Eq. (4.23) with $q_{3} \in \mathcal{F}_{0}$ along with the definitions (4.24) and (4.25). Let us denote it by

$$
\mathcal{B}:=\mathcal{F}_{1} \oplus \mathcal{V}_{a}
$$

We are now ready to state the main step towards the proof of Theorem 4.1.

Proposition 4.4. There is a homomorphism ı from the associative algebra $\mathcal{B}$ into the algebra of Hilbert-Schmidt operators over some real separable Hilbert space, such that

$$
c^{(n)}\left(q_{1}, \ldots, q_{n}\right)=\operatorname{Tr}\left(\iota\left(q_{1}\right) \cdots \iota\left(q_{n}\right)\right),
$$

and $\iota(\mathcal{F})$ are symmetric operators while $\iota\left(\mathcal{V}_{a}\right)$ are antisymmetric.

We shall give the proof of this proposition in the subsequent subsection. The main reason leading to it is that $\mathcal{B}$ becomes a real Hilbert algebra with an integral trace on it. Here we proceed to show how Theorem 4.1 can be proven by using the above results.

Proof of Theorem 4.1. Let $\Phi_{m}=\Phi_{m}^{0}+\Phi_{m}^{1}$ be the decomposition of each field $\Phi_{m}$ according to the splitting $\mathcal{F}=\mathcal{F}_{0} \oplus \mathcal{F}_{1}$. Take an orthonormal basis $\psi_{m}$ in $\mathcal{F}_{0}$ and let $\Phi_{m}^{0}=\sum_{j=1}^{\infty} \alpha_{m, j} \psi_{j}$, and $\beta_{m}=\left(\beta_{m, j, k}\right)_{j, k}$ be the symmetric matrix corresponding to the Hilbert-Schmidt operator $\iota\left(\Phi_{m}^{1}\right)(m=1,2, \ldots)$. Then Eqs. (4.3) and (4.27) show that the constants $\alpha_{m, j}$ and $\beta_{m, j, k}$ so defined satisfy the conditions of Theorem 4.1.

Remark 4.1. In general, we have $\mathcal{F}_{1} \supsetneqq \mathcal{V}_{s}$. This is because the elements of $\mathcal{F}_{1}$ correspond, by Proposition 4.4, to Hilbert-Schmidt symmetric operators and on the other hand, the elements of $\mathcal{V}$ are obtained, according to the inductive construction of Sect. 4.1, as products of elements of $\mathcal{F}$ and will, hence, correspond to trace class operators.

4.3. Completion of the proofs. It remains to prove Proposition 4.4. We start with an inequality of Cauchy-Schwartz type.

\section{Lemma 4.5.}

Let $q_{1}, q_{2} \in \mathcal{A}$ be such that each of them belongs either to $\mathcal{F}$ or to $\mathcal{V}_{a}$. Then we have

$$
\left\langle q_{1} * q_{2}, q_{1} * q_{2}\right\rangle^{2} \leqslant\left\langle q_{1} * q_{1}, q_{1} * q_{1}\right\rangle\left\langle q_{2} * q_{2}, q_{2} * q_{2}\right\rangle .
$$

Proof. Consider $\left\langle q_{1} * q_{1}+\lambda q_{2} * q_{2}, q_{1} * q_{1}+\lambda q_{2} * q_{2}\right\rangle \geqslant 0$ and use that $\left\langle q_{1} * q_{1}, q_{2} * q_{2}\right\rangle=$ $\pm\left\langle q_{1} * q_{2}, q_{1} * q_{2}\right\rangle$ if each of $q_{1}, q_{2}$ belongs either to $\mathcal{F}$ or to $\mathcal{V}_{a}$. 
The space $\mathcal{B}$ (4.26) is a real pre-Hilbert space with a scalar product given by (4.22). It is also invariant under the action of $t$ (actually the eigenspaces of $t$ are $\mathcal{F}_{1}$ and $\mathcal{V}_{a}$ ). The left action of $\mathcal{B}$ on itself gives us an algebra homomorphism

$$
\iota: \mathcal{B} \rightarrow \operatorname{Lin}_{\mathbb{R}} \mathcal{B}
$$

of $\mathcal{B}$ into the algebra of all operators over $\mathcal{B}$. Moreover, the elements of $\mathcal{F}$ are mapped into symmetric operators and the elements of $\mathcal{V}_{a}$, into antisymmetric (this is due to (4.23)).

\section{Lemma 4.6. Every element of $\mathcal{B}$ is mapped into a Hilbert-Schmidt operator.}

Proof. Since $\mathcal{B}$ is generated by $\mathcal{F}_{1}$ (according to the inductive construction of $\mathcal{F}$ and $\mathcal{V}$ in Sect. 4.1) it is enough to show this for the elements of $\mathcal{F}_{1}$.

Let $\phi \in \mathcal{F}_{1}$ and consider the commutative subalgebra $\mathcal{B}_{\phi}$ of $\mathcal{B}$ generated by $\phi$. The algebra $\mathcal{B}_{\phi}$ is freely generated by $\phi$, i.e., is isomorphic to the algebra $\lambda \mathbb{R}[\lambda]$ of polynomials in a single variable $\lambda(\leftrightarrow \phi)$, since $\phi$ belongs to the orthogonal complement of $\mathcal{F}_{0}$ (4.24). For a $p(\lambda) \in \lambda \mathbb{R}[\lambda]$ we shall denote by $\phi^{[p]}$ the corresponding element of $\mathcal{B}_{\phi}$. In particular,

$$
\phi^{\left[p_{1}\right]} * \phi^{\left[p_{2}\right]}=\phi^{\left[p_{1} p_{2}\right]} .
$$

Setting

$$
\phi^{*(n+1)}:=\phi^{* n} * \phi, \quad c\left[\lambda^{n+1}\right]:=c^{(2)}\left(\phi^{* n}, \phi\right) \equiv\left\langle\phi^{* n}, \phi\right\rangle
$$

$\left(\phi^{* 1}:=\phi, n \geqslant 1\right)$ we obtain a positive definite functional over the algebra $\lambda^{2} \mathbb{R}[\lambda] \cong$ $\phi * \mathcal{B}_{\phi}$ (due to Eq. (4.23) and the positivity of $\langle\cdot, \cdot\rangle(4.22)$ ).

Then, by the Hamburger theorem about the classical moment problem ([9, Chap. 12, Sect. 8]) we conclude that there exists a bounded positive Borel measure $\mathrm{d} \mu(\lambda)$ on $\mathbb{R}$, such that

$$
c\left[\lambda^{2} p(\lambda)\right]=\int_{\mathbb{R}} p(\lambda) d \mu(\lambda)
$$

for every $p(\lambda) \in \mathbb{R}[\lambda]$. Using this we can extend the fields $\phi^{[p]}(\mathrm{x})$ to $\phi^{[f]}(\mathrm{x})$ for Borel measurable functions $f$ having compact support with respect to $\mu$ in $\mathbb{R} \backslash\{0\}$. The latter can be done in the following way. Fix $\varepsilon \in(0,1)$ and let $g_{1}, \ldots, g_{n}$ be Schwartz test functions on $M$. By Theorem 4.2 the correlators $\left\langle 0\left|\phi^{\left[p_{1}\right]}\left[g_{1}\right] \cdots \phi^{\left[p_{n}\right]}\left[g_{n}\right]\right| 0\right\rangle$ depend polynomially on $c^{(n)}\left(\phi^{\left[p_{k_{1}}\right]}, \ldots, \phi^{\left[p_{k_{j}}\right]}\right)=c\left[p_{k_{1}} \cdots p_{k_{j}}\right]$ for all $\left\{k_{1}, \ldots, k_{j}\right\} \subseteq\{1, \ldots, n\}$. But for every $\varepsilon \in(0,1)$ there exists a norm

$$
\|q\|_{\varepsilon}=A_{\varepsilon} \sup _{|\lambda| \leqslant \varepsilon}\left|\frac{q_{k}(\lambda)}{\lambda^{2}}\right|+B_{\varepsilon} \int_{\mathbb{R} \backslash(-\varepsilon, \varepsilon)}\left|q_{k}(\lambda)\right| d \mu(\lambda)
$$

on $\lambda^{2} \mathbb{R}[\lambda] \ni q(\lambda)$, where $A_{\varepsilon}$ and $B_{\varepsilon}$ are some positive constants, such that for every $q_{1}, \ldots, q_{m} \in \lambda^{2} \mathbb{R}[\lambda]$,

$$
\left|c\left[q_{1}(\lambda) \cdots q_{m}(\lambda)\right]\right| \leqslant \prod_{k=1}^{m}\left\{\int_{\mathbb{R}} \frac{\left|q_{k}(\lambda)\right|^{m}}{|\lambda|^{2}} d \mu(\lambda)\right\}^{\frac{1}{m}} \leqslant \prod_{k=1}^{m}\left\|q_{k}\right\|_{\varepsilon} .
$$


Hence, $\left|\left\langle 0\left|\phi^{\left[p_{1}\right]}\left[g_{1}\right] \cdots \phi^{\left[p_{n}\right]}\left[g_{n}\right]\right| 0\right\rangle\right| \leqslant C \prod_{k=1}^{n}\left\|p_{k}\right\|_{\varepsilon}\left\|g_{k}\right\|_{S}$ for some constant $C$ and Schwartz norm $\|\cdot\|_{S}$ (not depending on $p_{k}$ and $g_{k}$ ). Since for every $\varepsilon \in(0,1)$ the Banach space $L^{1}(\mathbb{R} \backslash\{(-\varepsilon, \varepsilon)\}, \mu)$ is contained in the completion of $\lambda^{2} \mathbb{R}[\lambda]$ with respect to the norms (4.33), we can extend the linear functional $c[p(\lambda)]$ as well as the correlators $\left\langle 0\left|\phi^{\left[p_{1}\right]}\left[g_{1}\right] \cdots \phi^{\left[p_{n}\right]}\left[g_{n}\right]\right| 0\right\rangle$ to a functional $c[f(\lambda)]$ and correlators $\langle 0| \phi^{\left[f_{1}\right]}\left[g_{1}\right] \cdots$ $\phi^{\left[f_{n}\right]}\left[g_{n}\right]|0\rangle$ defined for Borel functions $f, f_{1}, \ldots, f_{n}$ compactly supported with respect to $\mu$ in $\mathbb{R} \backslash\{0\}$. Thus, we can extend the fields $\phi^{[p]}$ by extending their correlators.

By the continuity we also have for arbitrary Borel functions $f, f_{k}$, compactly supported in $\mathbb{R} \backslash\{0\}$ :

$$
\begin{gathered}
\phi^{\left[f_{1}\right]} * \phi^{\left[f_{2}\right]}=\phi^{\left[f_{1} f_{2}\right]}, \quad c^{(n)}\left(\phi^{\left[f_{1}\right]}, \ldots, \phi^{\left[f_{n}\right]}\right)=c\left[f_{1} \cdots f_{n}\right], \\
c[f]=\int_{\mathbb{R}} \frac{f(\lambda)}{\lambda^{2}} d \mu(\lambda)
\end{gathered}
$$

(cp. (4.32)), and $c^{(n)}$ determine the correlation functions of $\phi^{\left[f_{k}\right]}$ as in Theorem 4.2.

In particular, for every characteristic function $\chi_{S}$ of a compact subset $S \subset \mathbb{R} \backslash\{0\}$ we have $\phi^{\left[\chi_{S}\right]} * \phi^{\left[\chi_{S}\right]}=\phi^{\left[\chi_{S}\right]}$. Hence, for such a $d=2$ field we will have that all its truncated correlation functions are given by (4.2) with all normalization constants $c^{(n)}$ equal to one and the same value $c^{(2)}\left(\phi^{\left[\chi_{s}\right]}, \phi^{\left[\chi_{s}\right]}\right)$. Then, as shown in [12, Theorem 5.1], Wightman positivity requires this value to be a non-negative integer, i.e.,

$$
c^{(2)}\left(\phi^{\left[\chi_{S}\right]}, \phi^{\left[\chi_{S}\right]}\right)=c\left[\chi_{S}\right]=\int_{S} \frac{d \mu(\lambda)}{\lambda^{2}} \in\{0,1,2, \ldots\}
$$

(it is zero iff $\phi^{\left[\chi_{S}\right]}=0$ ). Hence, the restriction of the measure $d \mu(\lambda) / \lambda^{2}$ to $\mathbb{R} \backslash\{0\}$ is a (possibly infinite) sum of atom measures of integral masses, each supported at some $\gamma_{k} \in \mathbb{R} \backslash\{0\}$ for $k=1, \ldots, N$ (and $N$ could be infinity). In particular, the measure $\mu$ is supported in a bounded subset of $\mathbb{R}$.

By Lemma 4.5 we can define $\iota\left(\phi^{[f]}\right)$ as a closable operator on $\mathcal{B}$ if $f$ is a Borel measurable function with compact support in $\mathbb{R} \backslash\{0\}$. It follows then that the projectors $\iota\left(\phi^{\left[\chi_{s}\right]}\right)$, for a compact $S \subseteq \mathbb{R} \backslash\{0\}$, provide a spectral decomposition for $\iota(\phi)$ (in fact, $\left.\iota\left(\phi^{[f]}\right)=f(\iota(\phi))\right)$. Thus, $\iota(\phi)$ has discrete spectrum with eigenvalues $\gamma_{k}(k \in \mathbb{N})$, each of a multiplicity given by the integer $c^{(2)}\left(\phi^{\chi\left\{\gamma_{k}\right\}}, \phi^{\chi\left\{\gamma_{k}\right\}}\right)$. Then $\iota(\phi)$ is a Hilbert-Schmidt operator since

$$
\sum_{k=1}^{\infty} \gamma_{k}^{2} c^{(2)}\left(\phi^{\chi_{\left\{\gamma_{k}\right\}}}, \phi^{\chi\left\{\gamma_{k}\right\}}\right)=\sum_{k=1}^{\infty} \gamma_{k}^{2} \int_{\left\{\gamma_{k}\right\}} \frac{d \mu(\lambda)}{\lambda^{2}}=\int_{\mathbb{R} \backslash\{0\}} d \mu(\lambda)<\infty
$$

( $\mu$ being a bounded measure).

The completion of the proof of Proposition 4.4 is provided now by the following corollary.

Corollary 4.7. For every $q_{1}, q_{2} \in \mathcal{B}$ one has $c^{(2)}\left(q_{1}, q_{2}\right)=\operatorname{Tr} \quad\left(\iota\left(q_{1}\right) \iota\left(q_{2}\right)\right)$.

Proof. If $q_{1}=q_{2} \in \mathcal{F}_{1}$ this follows from the proof of Lemma 4.6 and hence, by a polarization, for any $q_{1}, q_{2} \in \mathcal{F}_{1}$. The general case can be obtained by using the facts that $\mathcal{B}$ is generated by $\mathcal{F}_{1}$ and $c^{(2)}$ has the symmetry $c^{(2)}\left(q_{1} * q_{2}, q_{3}\right)=c^{(2)}\left(q_{1}, q_{2} * q_{3}\right)$. 


\section{Discussion. Open Problems}

The main result of Sect. 4, the (generalized) free field representation of a system $\left\{\phi_{a}\right\}$ of GCI scalar fields of conformal dimension $d=2$ (Theorem 4.1), is obtained by revealing and exploiting a rich algebraic structure in the space $\mathcal{F} \times \mathcal{V}$ of all $d=2$ real scalar fields and of all harmonic bilocal fields of dimension $(1,1)$. However, this structure is mainly due to the fact that we are in the case of lower scaling dimension: there is only one possible singular structure in the OPE (after truncating the vacuum part). One can try to establish such a result in spaces of spin-tensor bilocal fields (of dimension $\left(\frac{3}{2}, \frac{3}{2}\right)$ or $(2,2)$ ) satisfying linear (first order) conformally invariant differential equations (that again imply harmonicity). If these equations together with the corresponding pole bounds imply such singularities in the OPE, which can be "split" one would be able to prove the validity of free field realizations in such more general theories, too.

One may also attempt to study models, say in a theory of a system of scalar fields of dimension $d=4$, without leaving the realm of scalar bilocal harmonic fields $V_{1}$ (of dimension $(1,1))$. In [11] there have been found examples of 6-point functions of harmonic bilocal fields, which do not have free field realizations. However, our experience with the $d=2$ case shows that in order to complete the model (including the check of Wightman positivity for all correlation functions) it is crucial to describe the OPE in terms of some simple algebraic structure (e.g., associative, or Lie algebras).

On the other hand going beyond bilocal $V_{1}$ 's is a true signal of nontriviality of a GCI model. Our analysis of Sect. 3 shows that this can be characterized by a simple property of the correlation functions: the violation of the single pole property (of Sect. 3.3). From this point of view a further exploration of the example of Sect. 3.5 within a QFT involving currents appears particularly attractive.

Note added in proof: In [15], we have determined the biharmonic function whose leading part is given by Eq. (3.31). It involves dilogarithmic functions, whose arguments are algebraic functions of conformal cross ratios. This exemplifies the violation of Huygens bilocality for the biharmonic fields, Theorem 3.7. Yet, in support of Conjecture 3.3, it is shown that the structure of the cuts is in a nontrivial manner consistent with ordinary bilocality.

Acknowledgement. We thank Yassen Stanev for an enlightening discussion. This work was started while N.N. and I.T. were visiting the Institut für Theoretische Physik der Universität Göttingen as an Alexander von Humboldt research fellow and an AvH awardee, respectively. It was continued during the stay of N.N. at the Albert Einstein Institute for Gravitational Physics in Potsdam and of I.T. at the Theory Group of the Physics Department of CERN. The paper was completed during the visit of N.N. and I.T. to the High Energy Section of the I.C.T.P. in Trieste, and of K.-H.R. at the Erwin Schrödinger Institute in Vienna. We thank all these institutions for their hospitality and support. N.N. and I.T. were partially supported by the Research Training Network of the European Commission under contract MRTN-CT-2004-00514 and by the Bulgarian National Council for Scientific Research under contract PH-1406.

Open Access This article is distributed under the terms of the Creative Commons Attribution Noncommercial License which permits any noncommercial use, distribution, and reproduction in any medium, provided the original author(s) and source are credited.

\section{References}

1. Bakalov, B., Nikolov, N.M.: Jacobi identity for vertex algebras in higher dimensions. J. Math. Phys. 47, 053505 (2006)

2. Bakalov, B., Nikolov, N.M., Rehren, K.-H., Todorov, I.: Unitary positive-energy representations of scalar bilocal quantum fields. Commun. Math. Phys. 271, 223-246 (2007) 
3. Bargmann, V., Todorov, I.T.: Spaces of analytic functions on a complex cone as carriers for the symmetric tensor representations of $S O(N)$. J. Math. Phys. 18, 1141-1148 (1977)

4. Borchers, H.-J.: Über die Mannigfaltigkeit der interpolierenden Felder zu einer interpolierenden S-Matrix. N. Cim. 15, 784-794 (1960)

5. Buchholz, D., Mack, G., Todorov, I.T.: The current algebra on the circle as a germ of local field theories. Nucl. Phys. B (Proc. Suppl.) 5, 20-56 (1988)

6. Dobrev, V.K. Mack, G., Petkova, V.B., Petrova, S.G., Todorov, I.T.: Harmonic Analysis of the n-Dimensional Lorentz, Group and Its Applications to Conformal Quantum Field Theory. Berlin et al.: Springer, 1977

7. Dolan, F.A., Osborn, H.: Conformal four point functions and operator product expansion. Nucl. Phys. B 599, 459-496 (2001)

8. Dütsch, M., Rehren, K.-H.: Generalized free fields and the AdS-CFT correspondence. Ann. H. Poincaré 4, 613-635 (2003)

9. Dunford, N., Schwartz, J.: Linear Operators, Part 2. Spectral Theory. Self Adjoint Operators in Hilbert Space. N.Y.-London: Interscience Publishers, 1963

10. Mack, G.: All unitary representations of the conformal group $S U(2,2)$ with positive energy. Commun. Math. Phys. 55, 1-28 (1977)

11. Nikolov, N.M., Rehren, K.-H., Todorov, I.T.: Partial wave expansion and Wightman positivity in conformal field theory. Nucl. Phys. B 722, 266-296 (2005)

12. Nikolov, N.M., Stanev, Ya.S., Todorov, I.T.: Four dimensional CFT models with rational correlation functions. J. Phys. A 35, 2985-3007 (2002)

13. Nikolov, N.M., Stanev, Ya.S., Todorov, I.T.: Globally conformal invariant gauge field theory with rational correlation functions. Nucl. Phys. B 670, 373-400 (2003)

14. Nikolov, N.M., Todorov, I.T.: Rationality of conformally invariant local correlation functions on compactified Minkowsi space. Commun. Math. Phys. 218, 417-436 (2001)

15. Nikolov, N.M., Rehren, K.-H., Todorov, I.: Pole structure and biharmonic fields in conformal QFT in four dimensions. In: Dobrev, V. (ed.) LT7: Lie Theory and its Applications in Physics, Proceedings Varna 2007, Sofia, Heron Press (2008). e-print arXiv:0711.0628, to appear

16. Schroer, B., Swieca, J.A.: Conformal transformations of quantized fields. Phys. Rev. D 10, 480-485 (1974)

17. Schroer, B., Swieca, J.A., Völkel, A.H.: Global operator expansions in conformally invariant relativistic quantum field theory. Phys. Rev. D 11, 1509-1520 (1975)

18. Streater R.F., Wightman A.S.: PCT, Spin and Statistics, and All That. Benjamin, 1964; Princeton, N.J.: Princeton Univ. Press, 2000

19. Todorov, I.: Vertex algebras and conformal field theory models in four dimensions. Fortschr. Phys. 54, 496-504 (2006)

Communicated by Y. Kawahigashi 